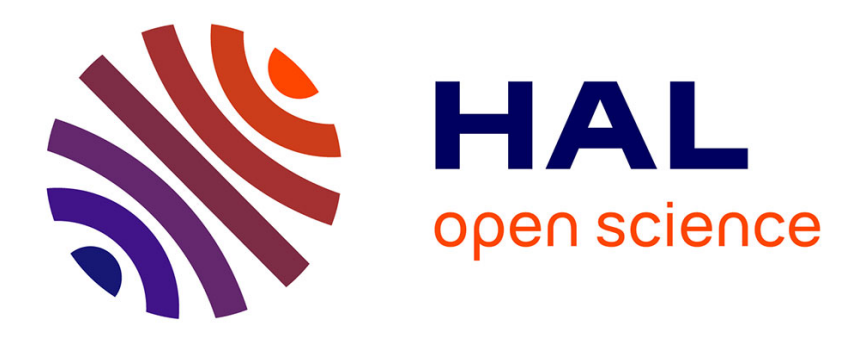

\title{
La délibération des images. Vers une nouvelle pragmatique du cinéma et de l'audiovisuel
}

\author{
Guillaume Soulez
}

\section{To cite this version:}

Guillaume Soulez. La délibération des images. Vers une nouvelle pragmatique du cinéma et de l'audiovisuel. Communication \& langages, 2013, 2013 (176), pp.3-32. 10.4074/S0336150013012015 . hal-01366344

\section{HAL Id: hal-01366344 \\ https://hal.science/hal-01366344}

Submitted on 14 Sep 2016

HAL is a multi-disciplinary open access archive for the deposit and dissemination of scientific research documents, whether they are published or not. The documents may come from teaching and research institutions in France or abroad, or from public or private research centers.
L'archive ouverte pluridisciplinaire HAL, est destinée au dépôt et à la diffusion de documents scientifiques de niveau recherche, publiés ou non, émanant des établissements d'enseignement et de recherche français ou étrangers, des laboratoires publics ou privés. 
La délibération des images. Vers une nouvelle pragmatique du cinéma et de l'audiovisuel $^{1}$

Des romans, des films, des émissions de télévision, nous ne cessons de discuter : ne pourrions-nous pas faire de cette donnée culturelle et sociale un point de départ pour repenser notre modèle d'analyse de la communication, et de la communication audiovisuelle en particulier ? Il est souvent difficile de " socialiser » nos modèles sémiotiques. Intégrer les modes de raisonnement et de sémiotisation propres à la discussion publique pour l'analyse des films permet de saisir que la relation entre films (au sens large) et " espace public », ou sphère publique, ne se réduit pas à la seule identification des thèmes de ces films, ou au fait que les films sont des objets qui circulent dans l'espace public et dont on se sert pour délibérer. En effet, si on raisonne ainsi, on risque de séparer le moment sémiotique du moment délibératif : regarder le film relèverait de la sémiotique, en discuter ensuite, de la discussion politique. Il serait plus juste de considérer que le spectateur ne met pas au vestiaire son costume de citoyen en entrant dans la salle de cinéma ou en s'asseyant sur son canapé : il existe une dynamique sémiotique spécifique à notre immersion dans un espace

1. Cet article a pour origine une conférence donnée au CELSA le 13 juin 2012. J'en remercie les organisateurs Yves Jeanneret, Emmanuël Souchier et Adeline Wrona, qui m'ont donné l'occasion de présenter les grandes lignes d'un ouvrage (achevé fin 2008, paru en 2011) et les pistes de travail qui sont issues du croisement de ces recherches sur la " lecture rhétorique " au cinéma et à la télévision avec d'autres sur le Service de la recherche de l'ORTF, la question du " dispositif » et la réception. Je remercie également Daniel Dayan, Jostein Gripsrud ainsi que mes collègues de l'université Paris 3, notamment Roger Odin et Laurent Jullier, pour leurs remarques à l'occasion de discussions sur cette approche.

\section{GUILLAUME SOULEZ}

Cet article étudie la façon dont les spectateurs, en tant que membres d'un espace de discussion publique, s'emparent des films et documents audiovisuels pour mettre en débat les images et les sons. Comprendre cette " délibération des images " suppose de revenir sur la relation entre rhétorique et images pour mieux identifier une capacité plus générale de discussion chez les spectateurs qui lisent les images comme arguments et points de vue par rapport à des enjeux sociaux et politiques, mais qui les lisent aussi en tant qu'elles proposent, ou non, des ré-agencements par rapport aux attentes conventionnelles (genres audiovisuels par exemple), voire une éventuelle interrogation sur le simulacre lui-même. Les lieux et les niveaux de la délibération nous conduisent à une meilleure compréhension du rapport entre délibération, réception et production de formes.

Mots-clés : cinéma, télévision, audiovisuel, médias, Rhétorique, poétique, délibération, pragmatique, espace public, réception 
public qui se manifeste, sous des formes diverses qu'il importe de préciser, pendant la lecture des films.

On propose donc ici quelques pistes pour une "délibération des images » en revenant tout d'abord sur la question des relations entre la tradition rhétorique de l'étude du discours public et l'analyse des images pour éclairer quelques points théoriques préalables, afin de mieux souligner l'apport d'un usage pragmatique de la perspective rhétorique dans l'étude de films et documents audiovisuels. Ceci permet d'aboutir à l'identification d'une « lecture rhétorique des images et des sons ", dont nous avons proposé la mise au point dans Quand le film nous parle. Rhétorique, cinéma, télévision ${ }^{2}$, par opposition à la "lecture poétique ", et dont nous allons préciser quelques aspects ici. La deuxième étape de ce questionnement débouche sur la prise en compte de l'existence d'une discussion publique qui porte non plus sur les enjeux sociaux et politiques directement accessibles par le « discours du film», mais sur les questions « poétiques » elles-mêmes. Quelle place faire à ce questionnement, s'agit-il d'un élargissement du domaine de la rhétorique ou d'un changement de la perspective mettant en valeur une capacité plus générale de discussion du spectateur-auditeur ? On défendra la seconde hypothèse en détaillant les différents niveaux de la délibération spectatorielle, de l'ancrage sociopolitique à l'activité sémiotique elle-même, et réciproquement, pour mettre au cour de la réflexion l'articulation entre production de sens et sphère publique à l'endroit du film ou du document audiovisuel. On développera enfin quelques pistes pour préciser cette démarche en lien avec les questions de " réception » et d'analyse des « formes » audiovisuelles, qui sont l'horizon de cette recherche.

\section{DE LA « RHÉTORIQUE ॥ À LA dÉLIBÉRATION}

Sans entrer dans le détail de la démonstration que nous avons proposée par ailleurs, la première étape nécessaire d'une démarche fondée sur l'hypothèse d'une continuité entre notre ancrage comme spectateurs dans une sphère publique et notre appréhension des films et documents audiovisuels est certainement le ré-examen lucide des travaux qui ont proposé d'analyser les images avec le concours de la tradition rhétorique, depuis le célèbre article fondateur de Roland Barthes, "Rhétorique de l'image ${ }^{3}$, source de nombreux malentendus. Sa diffusion académique, sociale et pédagogique, sous une forme souvent simplifiée, explique une bonne part des difficultés actuelles à faire émerger une autre perspective sur les liens entre images et espace public dans le champ de l'étude du cinéma et des médias audiovisuels. Comme Barthes l'a reconnu lui-même ${ }^{4}$, mais d'autres aussi (comme Jean-Marie Klinkenberg du Groupe $\mu^{5}$ ), la jonction dans les années 1960 entre sémiologie/sémiotique et tradition rhétorique s'est faite, sous l'influence du travail de Jakobson sur métaphore et métonymie, au niveau d'un petit segment de cette tradition, l'elocutio, dans sa version latine tardive, revue ensuite par des siècles de théorie littéraire dite "classique », aboutissant à cette " restriction » assumée par

2. Guillaume Soulez, Quand le film nous parle. Rhétorique, cinéma, télévision, PUF, 2011.

3. Roland Barthes, "Rhétorique de l'image », Communications, 4, 1964, p. 40-51.

4. Roland Barthes, «La Rhétorique ancienne : aide-mémoire », Communications, 16, 1970, p. 254-340.

5. Jean-Marie Klinkenberg, «Rhétorique de l'argumentation et Rhétorique des figures », in Alain Lempereur et Michel Meyer (éd.), Figures et conflits rhétoriques, Université de Bruxelles, 1990, p. 115-137. 
Genette autour des «figures » dites « de rhétorique " ${ }^{6}$. En réalité, au moment où se développait la pragmatique anglaise et américaine avec Austin puis Searle, c'est une version " poétique » de la Rhétorique, une version littéraire de l'« efficacité » des figures, une version de la Rhétorique digérée par une théorie poétique littéraire en plein rebond, qui a servi à Barthes et à ceux qui l'ont suivi à rendre raison de la nouvelle « culture de masse » visuelle qui émerge avec le cinéma, la télévision et la publicité. Comme on sait, est « rhétorique » pour Barthes la connotation implicite du message publicitaire qui circule sous l'évidence dénotative et apparemment objective de la photographie.

Renforcée par l'hypothèse puissante d'un " discours du récit » (le discours étant implicite et souterrain, là où le récit est " naturel » et apparent), la sémiologie de l'image est très largement encore aujourd'hui une poétique de l'image, ce qui s'est trouvé en particulier accentué dans le champ des études cinématographiques et audiovisuelles par l'intérêt porté par Christian Metz aux questions du récit en images et au développement académique de la narratologie audiovisuelle qui s'en est suivi. Or, comme le montrent à l'envi les études dites « de réception ", depuis qu'elles existent (qu'on pense notamment aux travaux bien connus de Lazarsfeld ou de Hoggart dans les années 1940-1950), les spectateurs (lecteurs, auditeurs) mobilisent bien une compétence discursive et des ressources argumentatives lorsqu'ils se positionnent par rapport aux images et sons de leur environnement médiatique, et peut-être en particulier quand ils sont aux prises avec cet environnement, quand ils se saisissent des images, des mots, des sons pour en tirer du sens. Il nous paraît donc impossible de continuer à analyser ces images comme si elles faisaient l'objet d'une immersion poétique du spectateur dans le récit en images (trop souvent confondue, d'ailleurs, avec son " aliénation »), tout en constatant, voire en soulignant, d'un autre côté, une sorte d'autonomie, souvent critique, du spectateur " en aval », en « réception », comme s'il n'y avait pas de solution de continuité entre les deux.

\section{De la " Rhétorique de l'image " à la « lecture rhétorique " des images (et des sons)}

Ce n'est pas un hasard si, dans des contextes aussi différents que la France et les États-Unis, ce sont les travaux sur le documentaire ou la télévision, en lien avec des traditions d'analyse du discours (politique) et d'étude de l'argumentation (publique), qui ont maintenu l'idée que la parole au moins, mais aussi le montage (ou le découpage en direct) et la scénographie pouvaient avoir une fonction rhétorique et argumentative ${ }^{7}$. La notion de « dispositif» a eu de ce point de vue un

6. Gérard Genette, «La Rhétorique restreinte », Communications, 16, 1970, p. 233-253.

7. Voir en particulier sur la télévision de plateau : Noël Nel, Le débat télévisé, Armand Colin, 1990 ; Guy Lochard et Jean-Claude Soulages, " L'image, faire voir la parole ", in Patrick Charaudeau (dir.), La Télévision. Les débats culturels. "Apostrophes », Didier Érudition, 1991. Voir aussi le passage sur la "forme rhétorique » dans le documentaire, définie en lien avec l'argumentation, dans l'ouvrage classique de David Bordwell et Kristin Thompson, L'art du film : une introduction, De Boeck, 1999, p. 527-537; ou, parmi d'autres exemples possibles, la réflexion du documentariste Frederick Wiseman sur l'« éloquence trouvée " (found eloquence) et sur son propre travail de montage et de structuration discursive : Ira Halberstadt, "An interview with Frederick Wiseman ", Filmakers Newsletter, 7(4), 1974, et Christopher 
rôle ambivalent, reconduisant le modèle sémio-psychique de la théorie poétique du cinéma des années 1970 (en prenant appui sur le modèle disciplinaire de Foucault pour montrer sa nature politique), tout en servant aussi, ultérieurement, de façon plus descriptive mais en réalité plus opératoire ${ }^{8}$, à montrer que la scénographie et les choix de filmage pouvaient avoir une fonction argumentative (en instaurant des rôles discursifs, par exemple). Parallèlement, les travaux sur les premiers temps du cinéma ont également mis en valeur à la même époque non seulement l'importance d'un cinéma parlé et adressé au spectateur d'une façon bien différente du régime fictionnel de l'intégration « classique » hollywoodienne du spectateur au récit, mais aussi le lien entre cette adresse publique et le contexte de réception des films ${ }^{9}$. La continuité entre le " bonimenteur » des premiers temps et le "présentateur » d'aujourd'hui, passant par la voice-over du commentaire documentaire, pouvait alors apparaître plus nettement, et, avec elle, devant l'importance de la parole à la télévision, la nécessité de mettre en jeu un niveau rhétorique de l'analyse pour étudier les relations entre paroles et images télévisuelles, en s'appuyant en particulier sur les questions de "crédibilité " grâce à la notion d'ethos de l'orateur public, tandis que la notion de pathos permettait de souligner la relation de construction réciproque des attentes morales et émotionnelles des publics et des orateurs, notions resituées dans le cadre d'espaces publics et médiatiques historiquement construits et attestables ${ }^{10}$.

Mais une partie du chemin seulement était parcourue : si la recherche sur le pathos mettait sur la voie d'une bascule pragmatique plus entière, soumettant l'orateur au regard du public, c'est l'analyse de la construction de l'ethos des médiateurs de télévision par la presse, se faisant l'écho des attentes en termes d'ethos d'une société donnée ${ }^{11}$, qui a permis d'envisager une pragmatique rhétorique plus radicale ${ }^{12}$, reprenant l'hypothèse de la sémiopragmatique de Roger Odin. Selon celle-ci, «le sens n'est pas dans le film » (selon une formule de l'anthropologue Sol Worth), mais est construit par le contexte ${ }^{13}$, comme nous l'ont

- Ricks et Frederick Wiseman, «Language and Documentary Film: A Conversation with Frederick Wiseman ", The Threepenny Review, 41, 1990, p. 9-10.

8. Guy Lochard, "Parcours d'un concept dans les études télévisuelles. Trajectoires et logiques d'emploi », Hermès, 25, 1999, p. 143-151.

9. Voir, notamment : André Gaudreault et Germain Lacasse (éd.), « Le bonimenteur de vues animées/The Moving Picture Lecturer », Iris, 22, 1996.

10. Guillaume Soulez, La médiation rhétorique. Propositions pour une analyse du présentateur comme orateur médiatique, Université de Metz, Thèse de doctorat sous la direction de N. Nel, 1998.

11. Il faut, par exemple, que se soit imposée l'idée d'une « objectivité » des journalistes (que l'on peut repérer dans les discours médiatiques eux-mêmes ou dans les courriers de lecteurs qui valident cette conception) pour qu'ensuite le mode de lecture de l'ethos des journalistes-présentateurs de télévision soit déterminé par cette attente d'«objectivité ", qu'on peut, par exemple, analyser en termes d'arété ( «vertu », « justesse ») ou de phronésis («sagesse », « équilibre», «bon sens») dans le langage rhétorique aristotélicien. La perspective a l'avantage d'éviter le «médiacentrisme » et la lecture de l'ethos de tel ou tel présentateur se fait donc en fonction de l'adéquation entre une performance hic et nunc d'orateur et une image sociale historiquement déterminée.

12. Guillaume Soulez, "Ethos, énonciation, média. Sémiotique de l’ethos », Recherches en communication, 18, 2002, p. 175-198.

13. Roger Odin, Cinéma et production de sens, Armand Colin, 1990. 
montré la pragmatique austinienne puis la théorie de la pertinence de Sperber et Wilson, qui la prolonge en renversant le point de vue du locuteur à l'auditeur grâce à un modèle inférentiel. C'est alors tout un pan de la tradition rhétorique elle-même qu'il faut prendre à revers, celle qui met en avant le locuteur (l'orateur) en considérant que le pouvoir du discours est son pouvoir («l'art de persuader» selon une formule courante), en soulignant à quel point la Rhétorique est une pensée pragmatique (comme l'a indiqué la philosophie du langage contemporaine qui reconnaît bien volontiers sa dette à l'égard d'Aristote), et qui intègre de façon qu'on dirait aujourd'hui " dialogique » le public dans l'étude de la production du discours ${ }^{14}$. C'est là qu'on peut trouver, mais comme dans un miroir inversé, l'« efficacité » du discours rhétorique, qui n'est autre que celle que nous lui prêtons dans une société et une culture données, et selon des règles de pertinence qui sont celles de notre sphère publique du moment. Du coup, la « lecture rhétorique » peut être définie comme une lecture qui traite les images et les sons en tant qu'ils proposent des positions, des arguments, et même parfois des preuves, tandis que le programme d'une pragmatique rhétorique est de se demander : quand y-a-t-il cette lecture rhétorique des images et des sons ? Quand le spectateur-auditeur mobilise-t-il une lecture rhétorique des images et des sons, plutôt qu'un autre type de lecture ${ }^{15}$, c'est-à-dire, plus exactement, quand mobilise-t-il des ressources rhétoriques et argumentatives pour appréhender des images et des sons? Pourquoi le fait-il, et comment le fait-il ?

On passe ici d'une analyse qui permet de rendre compte du rôle de " pivot " de l'animateur d'Apostrophes ${ }^{16}$, rôle visuel autant que verbal qui lui permet de s'interposer entre l'écrivain et les téléspectateurs, dans une sorte de triangle discursif qui correspond à son rôle d'intercesseur et de porte-parole du public (expliquant sans doute le succès personnalisé de l'émission), à une analyse qui permet de comprendre comment le même débat télévisé de second tour de la campagne présidentielle, le 2 mai 2012, peut donner lieu à des lectures si différentes en Une du Figaro et de Libération le lendemain, 3 mai 2012 (le pourquoi étant ici peu difficile à comprendre, le contexte politique et médiatique de l'événement nous étant aisément accessible). Le premier prélève dans l'émission de la veille un plan d'ensemble, chacun des deux protagonistes à un bout de la table, levant la main droite en parlant, les deux journalistes au milieu, David Pujadas tentant visiblement en levant les deux mains de calmer les deux candidats, tandis que la mention «Haute tension » vient s'inscrire sur la nappe de la table. Alors que Libération propose un montage en forme de split-screen, une sorte de champ-contre-champ simultané, que nous n'avons pas pu voir stricto sensu en regardant la télévision (plan impossible selon les consignes très précises de filmage

14. Guillaume Soulez, "Rhétorique, public et "manipulation" ", Hermès, 38, 2004, p. 89-95.

15. Par exemple, parmi d'autres possibles (lecture musicale ou plastique, notamment), la « lecture poétique », qu'on peut définir comme le fait de lire un film pour produire un monde cohérent, qui neutralise les éléments qui pourraient être discordants (trouver un acteur vieilli, reconnaître un lieu familier à l'écran) et repose principalement sur une logique mimétique (représentation des actions des hommes) obéissant elle-même à des conventions de la représentation.

16. Guy Lochard et Jean-Claude Soulages, «L'image, faire voir la parole », art. cit. 
d'un tel débat), mais que nous avons pu en quelque sorte «fabriquer » à mesure de l'émission, en prenant appui sur l'alternance des plans télévisuels, chacune des deux moitiés verticales de l'image étant occupée par un protagoniste, François Hollande à gauche, visage ouvert, regard un peu vers le bas, Nicolas Sarkozy, visage légèrement baissé et fermé, regardant vers le haut, avec le titre «Hollande préside le débat ». Quand on est un journal de gauche, soutenant Hollande, on construit cette lecture qui tire de la «tension » repérée par le confrère de droite l'idée plus précise d'une domination de l'un sur l'autre, résultat de la lecture discursive du débat télévisé par Libération, mais aussi, sans doute, par la plupart de ses lecteurs : la direction de regard ici fait véritablement le raccord entre les deux moitiés du cadre, à la manière d'une inscription visuelle de la lecture faite par le quotidien. Pour Libération, l'ethos de Hollande, pourrait-on dire, a été plus " présidentiel » que celui de Sarkozy (présenté comme « sur la défensive » dans la même Une), ce qui était mesurable, pour Libération, au discours verbal proposé, mais qui s'est $v u$ aussi à l'écran à travers leurs postures et leurs regards respectifs. La scénographie du Figaro préfère, elle, dupliquer le dispositif « neutre » ou impartial du débat télévisé, qui renvoie de façon institutionnelle à une forme d'égalité des candidats dans l'affrontement (quitte à le légender à sa manière), mettant l'accent sur la moindre maîtrise des journalistes (disparaissant complètement dans le choix de Libération, qui prend aussi la main sur le montage), ce qui permet peut-être au journal d'éviter de répondre à la question - rituelle dans la presse du lendemain du « gagnant» d'un tel débat.

Il ne s'agit pas ici de souligner, comme cela a souvent été fait, que, en régime ordinaire, les organes de presse et les éditorialistes font, « comme par hasard », les mêmes interprétations que leurs lecteurs. Ces quelques remarques sur les Unes du Figaro et de Libération montrent ici plutôt, d'une façon probablement exacerbée parce que liée à un contexte politique peu opaque, comment nos lectures des images et des sons sont déterminées par ce contexte et les places respectives des responsables d'un discours dans un espace public donné, mais elles soulignent surtout combien notre lecture est toujours orientée, non pas seulement par des prédéterminations, de quelque nature qu'elles soient (culturelles, idéologiques, etc.), mais par les personnes à qui nous faisons part de notre lecture (les lecteurs de Libération pour le quotidien de gauche, les lecteurs du Figaro pour le quotidien de droite). En un mot, le journal nous dit : "c'est ce que nous avons vu (ensemble), n'est-ce pas?», mais il est possible de ne pas être d'accord (pensons, par exemple, à un militant qui tomberait sur la Une du journal adverse dans un kiosque ou sur le Web).

L'avantage de ce changement de position de l'analyse est qu'il permet de prendre en compte la lecture rhétorique des images et des sons depuis les spectateurs (nous avons étudié ici les journalistes et graphistes de Libération et du Figaro en tant que spectateurs, qui plus est s'adressant à d'autres spectateurs), là où l'approche précédente tend à isoler des "strates » (une strate verbale, une strate visuelle), puis à décomposer ces éléments de l'intérieur, sans pouvoir se prononcer sur leur effectivité sur les spectateurs (résidu de l'approche top-down du dispositif au sens traditionnel). L'approche pragmatique permet, en partant de 
« réceptions » (nous reviendrons sur ce terme) attestées, de comprendre quand (et comment) les spectateurs font une lecture globale de l'émission (ou du film) en discursivisant les images et les sons en fonction de leurs ressources et orientations propres (singulières ou collectives) au moment de leur contact avec le document audiovisuel. Ceci permet de considérer tout élément de ce document comme susceptible d'une discursivisation : parole bien sûr, langage para-verbal, mais aussi cadrage, mouvement de caméra, montage, bruitage et musique, casting, etc. On peut définir la lecture rhétorique comme la discursivisation d'une séquence audiovisuelle considérée par le spectateur-auditeur comme (lui) tenant un discours explicite au sein d'une sphère publique donnée ${ }^{17}$.

Du coup, plutôt que de postuler une séparation entre le visuel, qui relèverait d'une poétique audiovisuelle incluant le « dispositif », et une dimension verbale, qui relèverait d'une rhétorique de la parole, nous pouvons souligner l'existence d'une lecture poétique de la parole (par exemple pour donner un sens aux dialogues entre personnages) et l'existence d'une lecture rhétorique des images et des sons (plutôt que d'une rhétorique de l'image). Au point que nous observons comment la réception de la dimension visuelle (et sonore) peut donner lieu à toutes sortes de réarrangements discursifs déterminés par la lecture (comme le montrent les choix d'image de Unes), au même titre que les réarrangements (sélection, recontextualisation, modulation, etc.) que l'on observe dans les échanges rhétoriques verbaux. Ainsi, les invariants - codes verbaux, visuels et sonores -, au lieu d'être seulement postulés par l'analyste (avec un postulat précisément d'efficacité), sont eux-mêmes pris dans la discursivisation, comme le montrent, pour les codes visuels du débat télévisé concernant le montage et la disposition spatiale, la reprise de la «neutralité » scénographique par le Figaro et le split-screen de Libération.

On peut faire la même analyse d'une séquence du film Une fermme est une femme (Godard, 1961), où l'on observe, lors d'une dispute conjugale entre deux personnages de profil à une table, le choix d'un panoramique continu gauche-droite/droite-gauche, plutôt que d'un champ/contre-champ, à la manière d'un match de tennis, qui met le spectateur en position d' " arbitre » et qui interroge aussi la position choisie par le responsable du discours du film vis-à-vis de la question de l'émancipation féminine. Le panoramique continu se termine en effet par un plan fixe sur Angela, le personnage féminin, qui « perd » la joute verbale et déclare en une forme de généralité qui peut s'adresser aisément aux spectateurs : " Pourquoi c'est toujours les femmes qui souffrent? », favorisant la discursivisation de la séquence, si elle n'était pas déjà engagée par le spectateur (en 1961, avec

17. Nous précisons " explicite » dans la mesure où, selon le modèle du " discours du récit », le discours (par exemple la leçon morale ou politique qu'on peut tirer d'une histoire qui punit ceux qui se sont pour une société donnée - mal comportés) demeure la plupart du temps implicite. Notre approche permet, en sens inverse, de donner une place aux épigraphes inaugurales, ou aux morales finales de la fable, qui sont, elles, explicites et permettent en général d'encadrer la lecture et d'attribuer une responsabilité discursive sans trop d'ambiguïté (qu'elle soit personnalisée ou doxique-proverbiale). Voir Guillaume Soulez, Quand le film nous parle. Rhétorique, cinéma, télévision, op. cit., 2011. 
l'émergence du féminisme, ou aujourd'hui, d'une autre façon sans doute, à un moment où les questionnements gender sont plus que jamais à l'ordre du jour).

\section{De la lecture rhétorique à la délibération}

Arrivé à ce stade, le théoricien est confronté, nous semble-t-il, à un problème intéressant : si le spectateur se met non seulement à reconnaître les conventions poétiques (ici audiovisuelles) - la poétique est une théorie de la reconnaissance, comme on sait -, mais à les évaluer voire à les critiquer, par exemple en considérant que la position d'arbitre proposée par "Godard ", vu comme le responsable du discours du film, est assez commode et lui (nous) évite de prendre clairement position en faveur de l'émancipation féminine (jugement mêlant des aspects de l'ethos et du logos attribué à Godard), alors le spectateur interroge non seulement les choix de "Godard » (ou du film) vis-à-vis du féminisme mais aussi les conventions cinématographiques et audiovisuelles (panoramique, champ/contre-champ) et leurs usages. Si l'on rapproche cette dynamique critique (au sens étymologique du terme) de discussions contemporaines sur la standardisation des films et des programmes, sur le "formatage ", etc., on mesure que la dimension poétique est donc elle-même un enjeu de l'appréciation et de la discussion spectatorielle. En un mot, la lecture poétique peut faire l'objet, à son tour, d'une lecture rhétorique, le spectateur étant en somme capable de se dédoubler. Et inversement : pensons au jugement poétique que nous pouvons porter aujourd'hui, avec le recul historique, sur les films de propagande des années 1910-1930, en reconnaissant des « codes » du film de propagande (c'est aussi une manière de considérer que nous ne sommes plus adressés en tant que spectateurs par ces films, parce que notre sphère publique n'est plus la même, donc de neutraliser la lecture rhétorique).

Nous voyons donc ici que nous devons changer de modèle de « dispositif »: le théâtre ou le cinéma dit "modernes » se sont appuyés sur la distanciation brechtienne pour essayer de penser la mise en crise d'un dispositif de représentation vu comme enveloppant et disciplinant le spectateur, en particulier grâce à l'illusion du quatrième mur (la Verfremdung rend alors " étrange » la Darstellung jusque-là naturalisée). Cela présuppose un metteur en scène capable de solliciter l'attention du spectateur en brisant l'illusion de la représentation. Il s'agit ensuite de permettre au spectateur d'agir dans le monde, par exemple s'il se rend compte, grâce au gestus de l'acteur ou à des allusions scénographiques au monde contemporain (imaginons la projection d'images de manifestations ouvrières actuelles sur le fond de la scène d'une représentation du Mariage de Figaro), que les rapports entre personnages sont des rapports de classe. Dans ce modèle (et ce récit d'un metteur en scène tout puissant qui agit sur le spectateur, dans le cadre d'une pensée du " choc », dont on sait qu'elle est elle-même inspirée de la poétique du montage eisensteinien), la distanciation semble, comme son nom l'indique d'ailleurs, un phénomène interne à la lecture poétique. Une avant-garde éclairée conscientise le public, jusque-là prisonnier des conventions théâtrales ou visuelles. Or, si tant est que ce modèle ait eu une efficacité réelle sur le public, il semble clair qu'il n'est pas adapté à la description des expériences et des compétences spectatorielles contemporaines que mettent en lumière les études 
de réception (voir notamment les travaux désormais classiques de Katz et Liebes pour la télévision ${ }^{18}$ ou de Staiger pour le cinéma ${ }^{19}$ ), mais aussi les pratiques contemporaines des spectateurs, aujourd'hui bien documentées ${ }^{20}$, les effets de conformisme, ou de conformisation, étant, par exemple, davantage le fait du groupe de réception lui-même que du dispositif médiatique dont il est fait usage ${ }^{21}$.

La lecture rhétorique nous est, en réalité, beaucoup plus " naturelle ", immédiate et spontanée, que la lecture poétique, qui fait l'objet d'un apprentissage, d'un «dressage » dès le plus jeune âge ${ }^{22}$ (nous apprenons ainsi, par exemple, à distinguer la "réalité » de la " fiction»). Ce que la théorie de la Verfremdung saisit à juste titre est donc le caractère profondément ancré, idéologique, mais aussi artificiel (il suffit d'un artifice inverse pour faire s'évaporer l'illusion) de la lecture poétique. Là où elle échoue, c'est dans l'idée que seul le metteur en scène est capable - démiurge un jour, démiurge toujours - de briser l'illusion poétique, alors même que nous ne cessons de le faire lorsque nous critiquons un certain nombre de conventions comme porteuses d'un discours politique ou culturel auquel nous nous opposons, ou lorsque nous débattons de l'usage de telle convention par tel(s) responsable(s) de discours.

Dès lors, il nous est possible de penser un rapport critique au dispositif, depuis un dehors du dispositif, qui serait le fait d'un spectateur et non le fruit d'une action interne du metteur en scène, soit qu'il s'agisse d'un positionnement culturel ou politique qui entraîne une critique du dispositif poétique (pour prendre un exemple simple : une analyse féministe du western classique comme exacerbation de la domination masculine, depuis les choix d'échelle de plan jusqu'au système des actions diégétiques, en passant par le regard des personnages masculins sur les femmes-objets), soit qu'il s'agisse d'une remise en cause du dispositif qui entraîne une prise de distance culturelle ou politique (une critique du documentaire d'avant le cinéma-direct, en voix-off surplombante - voix de Dieu, voix du maître -, qui le considère comme témoignant d'un regard bourgeois sur le peuple, d'un regard colonialiste sur les personnes filmées, ou d'un rapport paternaliste aux spectateurs). Ce n'est pas le lieu ici de développer une autre approche du dispositif possible en lien avec cette perspective, mais on peut simplement indiquer qu'à la fin des années 1960, c'est-à-dire avant les théories du dispositif des années 1970, Pierre Schaeffer, alors directeur du Service de la Recherche de l'ORTF, avait proposé une théorie du dispositif bien différente (autour de la notion de " dispositif de recherche »). Prenant en compte à la fois un souci opérationnel (production pour l'antenne) et les contraintes institutionnelles (propres à toute

18. Elihu Katz et Tama Liebes, The Export of Meaning. Cross-Cultural Readings of Dallas, Oxford University Press, 1990.

19. Janet Staiger, Interpreting Films. Studies in the Historical Receptions of American Cinema, Princeton University Press, 1992.

20. Laurent Jullier et Jean-Marc Leveratto, Cinéphiles et cinéphilie. Une histoire de la qualité cinématographique, Armand Colin, 2010.

21. Dominique Pasquier, Cultures lycéennes. La tyrannie de la majorité, Autrement, 2005.

22. Remarque de D. Dayan à propos de la différence entre « lecture rhétorique » et « lecture poétique », Paris, début octobre 2012. 
organisation), il s'agissait de faire varier les paramètres d'un genre audiovisuel (par exemple l'interview, le film dit " pour enfants », etc.), afin de sortir les producteurs comme les spectateurs de leurs habitudes, en s'appuyant sur l'expérimentation professionnelle pour les uns (créativité et recherche de nouvelles formes) et sur la réflexivité pour les autres (capacité à se connaître en tant que spectateur) ${ }^{23}$. Cette double attaque de la «standardisation » (pour reprendre un vocable de la théorie de l'industrie culturelle diffusée en France à l'époque par Edgar Morin) par le versant professionnel (critique de l'organisation) et le versant spectatoriel (critique des paresses culturelles) permet d'envisager proprement une capacité d'analyse du dispositif : de même qu'il vaut mieux, en bonne pragmatique, considérer qu'il n'y a pas un type de montage qui serait le "montage discursif » ${ }^{24}$, mais plutôt des discursivisations du montage ${ }^{25}$, de même on peut penser que le spectateur est capable de mesurer les agencements et réagencements d'un dispositif audiovisuel en tant que tel, par rapport aux agencements «standards» ou conventionnels attendus $^{26}$. C'est de fait l'un des moyens courants de mesurer son plaisir ou déplaisir de spectateur (apprécier le caractère innovant d'un dispositif audiovisuel pour nous présenter une romance ou une question sociale traditionnelles, ou, au contraire, protester contre le caractère "formaté ", dit-on aujourd'hui, de telle émission de plateau, ou de tel film grand public ou non ${ }^{27}$ ).

Ce jeu d'évaluation du dispositif montre donc non seulement que toute poétique est plus ou moins partagée (et pas seulement subie), mais qu'il existe une capacité critique du spectateur, aussi permanente et structurante que l'activité de reconnaissance poétique, que nous pourrions considérer soit comme une " rhétorique élargie » à la poétique, soit comme un aspect d'une forme de

23. Pierre Schaeffer, Machines à communiquer, tomes 1 et 2, Seuil, 1970 et 1972 ; Guillaume Soulez, «Parole captée, parole capturée : de Pierre Schaeffer aux années 70 », in François Jost (dir.), Années 70. La télévision en jeu, CNRS Éditions, 2005, p. 107-129.

24. Vincent Amiel, Esthétique du montage, Nathan, 2001.

25. Vincent Amiel prend l'exemple de Resnais pour illustrer le «montage discursif », mais le cinéma de Resnais est, précisément, fondé sur une participation active du spectateur. Comme le montre, de façon emblématique, Muriel ou Le temps d'un retour (1963), c'est le spectateur qui vient donner sens à la présence de la question algérienne dans le film (en commençant, sur le plan poétique, par combler les trous et finir les phrases des personnages), en discursivisant le moindre plan, le moindre enchaînement, voire le film dans le film, etc. Cette discursivisation est aisée, bien sûr, lorsque le contexte y pousse : le film est ainsi sorti peu après les accords d'Évian, mais le recul historique (lors d'une reprise vingt ans après, par exemple) peut, à un autre niveau, favoriser une certain forme d'explicitation des enjeux par la réception (voir par exemple : Guillaume Soulez, "L’Algeria tra dialogo e montaggio. Retorica di Muriel (1963)", in Marina Galetti (dir.), Jean Cayrol, Dalla Notte e Dalla Nebbia, Turin, Kaplan, 2010, p. 258-278).

26. Guillaume Soulez, «Format et dispositif. Nouvelles règles du jeu esthétique en régime industriel des images et des sons ", in Emmanuelle André, François Jost, Jean-Luc Lioult et Guillaume Soulez (éd.), Penser la création audiovisuelle. Cinéma, audiovisuel, multimédia, Publications universitaires de Provence, 2009, p. 253-262.

27. Un exemple parmi d'autres : "Western formaté et mollasson qui tente en vain de transposer le classique Witness aux grands espaces et aux élevages de moutons. Mais l'intégration de l'impitoyable tueur au sein de la communauté religieuse et la romance qui en découle n'apportent que clichés et niaiseries à tout va. » (USMarshallCahill, 4 mars 2012, sur http://forum.westernmovies.fr à propos de L'étranger du Montana, 2002). 
discussion des formes audiovisuelles qui aurait deux versants : l'un portant sur des enjeux propres à l'existence de ces objets dans un espace social donné (normes professionnelles, normes du goût, recherches et attentes d'objets nouveaux...), l'autre sur des enjeux sociaux et politiques qui traversent les objets audiovisuels (la lecture rhétorique). Il nous paraît plus sage (pour éviter de produire, à fronts renversés, une poétique restreinte à sa rhétorisation) et plus heuristique, comme nous allons le voir, de faire l'hypothèse d'une délibération des images (et des sons) qui aurait, au moins, ces deux dimensions, bien souvent associées l'une à l'autre, comme nous l'avons déjà aperçu plus haut : la mise en discussion d'un enjeu sociopolitique à travers des formes audiovisuelles et la mise en discussion d'un enjeu conventionnel (ou plus généralement formel) à partir des formes audiovisuelles elles-mêmes. Ceci suppose une capacité du spectateur, nous allons le voir, à mettre en débat sa propre lecture du film ou du document audiovisuel, à la situer par rapport à d'autres lectures.

\section{LA DÉLIBÉRATION}

La notion de délibération souligne, dans les divers champs dans lesquels elle apparaît (politique, justice, philosophie...), le fait que le travail d'élaboration du jugement s'inscrit non seulement dans une pratique de discussion publique où prévaut l'échange des points de vue, mais aussi dans le cadre d'une visée opérationnelle (c'est un trait que souligne bien par exemple l'opposition entre " voix consultative » et "voix délibérative » dans une assemblée). Ainsi, même si la délibération peut être, dans certains cas, " intérieure » - nous allons y revenir -, elle n'est pas une simple rêverie ou une méditation flottante sur le sens des choses, mais elle a un but qui est de fixer, au moins provisoirement, sinon le choix d'une action, du moins la manière d'appréhender tel ou tel enjeu ou tel objet offert à l'examen. De plus, en étudiant différents modèles délibératifs depuis les Grecs, Gary Remer distingue bien ainsi un modèle « conversationnel » de l'échange des points de vue (la discussion entre amis, l'échange personnel égalitaire et coopératif) d'un modèle " oratoire » - celui qui nous intéresse ici-, où l'échange des points de vue se fait dans le cadre d'une discussion publique généralement agonistique (c'est le sens aristotélicien, où bouleusis dérive de boulê, l'assemblée des citoyens $)^{28}$. Plus précisément, cette approche de la délibération s'oppose à une conception (ultra-)normative de l'espace public et de la discussion, celle de l'Agir communicationnel d'Habermas ${ }^{29}$, dans la mesure où la reconnaissance de la conflictualité fait partie du processus de discussion lui-même, avec ce qu'elle suppose d'asymétrie, de censure ou auto-censure (voir en particulier les travaux de Nancy Fraser et Chantal Mouffe qui critiquent le modèle habermassien ${ }^{30}$ ), mais aussi de mise en scène au sens littéral, dont les différents moyens d'expression et les

28. Gary Remer, "Two models of deliberation: Oratory and Conversation in Ratifying the Convention", Journal of Political Philosophy, 8, 2000, p. 39-64.

29. Jürgen Habermas, Théorie de l'Agir communicationnel, tomes 1 et 2, Fayard, 1987.

30. Nancy Fraser, "Rethinking the Public Sphere: A Contribution to the Critique of Actually Existing Democracy”, Social Text, 25/26, 1990, p. 56-80 ; Chantal Mouffe, The Democratic Paradox, Verso Books, 2000 . 
différents médias sont partie prenante, avec leurs spécificités et leurs conventions propres (l'espace public est à la fois une sphère publique - l'espace des arguments et une scène publique, comme le souligne Louis Quéré $\left.{ }^{31}\right)$.

\section{Délibération et interprétation en conflits}

Notre proposition est donc de lier étroitement pour l'analyse de la production de sens ce qu'on appelle couramment le « conflit des interprétations »- ou plus exactement l'existence d'interprétations en conflit, c'est-à-dire le désaccord que l'on peut exprimer plus ou moins publiquement avec d'autres interprétations d'un même phénomène ou d'une même production culturelle - avec le caractère conflictuel de la délibération. Selon cette perspective, la pluralité des lectures possibles d'un même film (générant ensuite des conflits), repérée par le sens commun et étudiée par les études de réception et certaines approches pragmatiques, ne permet pas de comprendre pourquoi c'est tel débat qui a lieu en réception. Il faut donc faire remonter le débat public au sein même de la lecture, au sein même du processus de sémiotisation. Pour travailler sur les relations entre espace public et document audiovisuel, je propose donc de considérer que cette pluralité et cette conflictualité sont un point de départ et non un point d'arrivée de l'analyse : le spectateur est alors un opérateur de cette pluralité et de cette conflictualité, et pas seulement un représentant d'une lecture potentielle parmi d'autres possibles ou un simple point de passage de déterminations socioculturelles. Or, c'est précisément ce que nous incite à considérer une sémiotique de l'espace public : à côté de sa capacité à reconnaître des codes, la pluralité des lectures est une composante fondamentale de l'activité sémiotique du spectateur en tant qu'il est membre d'un espace de discussion publique. Ainsi, côtoyant les modes de lecture déterminés par les habitudes culturelles (par exemple les grands genres audiovisuels), nous devons faire une place aux formes de relation aux discours et aux objets des discours qui croisent et parfois réajustent ou remettent en cause ces modes de lecture. En somme, le contexte socioculturel des habitudes de lecture (généralement intériorisées) est lui-même sur-déterminé par le contexte plus général de la discussion publique. Celle-ci est plus explicite et plus ouverte, car la discussion publique fait s'affronter ouvertement des sémiotisations différentes qui peuvent être, ou avoir été, en débat pour le spectateur lui-même au moment du film. Inversement, le travail spectatoriel peut donc littéralement avoir lieu parce que le spectateur rejoue en lui, au moment de produire du sens, cet antagonisme des points de vue différents sur l'objet qu'il considère. Ainsi, en quelque sorte, lorsqu'il délibère avec un film ou un document audiovisuel, le spectateur se fait espace public.

L'arrimage de l'analyse sémiotique à la délibération publique permet donc de donner un clair ancrage sociopolitique à l'activité sémiotique (un ancrage transcendental, au sens d'une condition de possibilité), plutôt que de chercher à retrouver dans le monde des signes des équivalents ou des transpositions du monde social, sans pour autant changer le modèle de l'activité sémiotique elle-même, qui reste surdéterminée par le modèle représentationnel (ou poétique), comme on

31. Louis Quéré, Les Miroirs équivoques, Aubier, 1982.

communication \& langages $-n^{\circ} 176-$ Juin 2013 
peut le voir de façon emblématique $\mathrm{e}^{32}$ dans la socio-sémiotique d'Eliseo Verón ${ }^{33}$. C'est, au contraire, précisément le projet d'un modèle sémiotique délibératif. Dès lors, la délibération dite "intérieure » (si tant est que cette expression ait un sens) est en réalité la scène de confrontation, chez un spectateur donné, de plusieurs interprétations possibles d'une séquence ou d'un objet ou d'un ensemble d'objets audiovisuels, en fonction des différentes attaches, attentes et projections culturelles (en termes de genres par exemple), sociales (en termes de distinction ou de désir d'appartenance à un collectif) et politiques (préférences politiques, position perçue comme dominée ou dominante, etc.) de ce spectateur ${ }^{34}$. Ce choix théorique a, bien sûr, des conséquences sur le plan politique car il s'agit de restituer aux acteurs sociaux que sont les spectateurs leur part d'agency à travers leur travail interprétatif, pour reprendre le terme de Stuart Hall (interpretative work), quand, sur la base d'un monde social vu comme lieu d'un « conflit culturel », il défend la vertu des études de réception au sein des Cultural Studies ${ }^{35}$. Cela nous permet, à la fois, d'éviter l'alternative habermassienne, trop normative (qui oppose l'usage stratégique et l'usage communicationnel de la discussion), en mettant au premier plan la dimension conflictuelle, mais aussi de complexifier dans l'analyse, du fait du caractère ouvert, pluriel et graduel de la délibération, l'alternative entre résistance et aliénation chez Hall.

Pour certains théoriciens de la délibération démocratique, c'est justement la capacité du spectateur à adopter d'autres points de vue que le sien à travers romans et films qui peut servir de modèle pour comprendre l'intégration du point de vue d'autrui, que ce soit en l'absence des interlocuteurs (comme dans la démocratie à la fois de masse et à distance), mais aussi en présence des partenaires de la délibération (par exemple lors d'une réunion), comme le souligne Robert Goodin $^{36}$. D'où la défense par ces auteurs du rôle fondamental des arts dans

32. «Emblématique » parce que l'effort remarquable d'Eliseo Verón de s'arracher du texto-centrisme et du modèle littéraire de Barthes (1964) pour défendre, en sens inverse, la part du corps et l'inscription des textes médiatiques dans des contextes anthropologiques, culturels et politiques, ne s'accompagne pas d'un changement équivalent pour l'analyse de l'activité sémiotique elle-même, malgré son enrichissement par la sémiotique peircienne (mais on trouve la même chose chez Umberto Eco).

33. Eliseo Verón, « De l'image sémiologique aux discursivités. Le temps d'une photo », Hermès, 13-14, 1994, p. 45-64.

34. Il faut donc connecter la théorie du spectateur au singulier (théorie du dispositif, théorie du cinéma) avec la théorie des spectateurs, au pluriel, ou théorie du «public " (réception, espace public, anthropologie culturelle). À partir d'un modèle dramaturgique et interactionniste, la notion $\mathrm{d}^{\prime}$ " audienciation » chez Daniel Dayan permet ainsi de comprendre comment un spectateur se rallie à un collectif (un "public ») en fonction de ses représentations du point de vue d'autrui sur le même objet médiatique (voir Daniel Dayan, «Le double corps du spectateur », in Jérôme Bourdon et François Jost (éd.), Penser la télévision, Ina-Nathan, 1998, p. 234-248), ce qui correspond bien à la dimension "scénique " de l'espace public. J'ai proposé pour ma part une version pragmatique de la théorie de l'« auditoire universel » de Perelman pour souligner sa dimension argumentative (voir Guillaume Soulez, " Nous sommes le public". Apports de la Rhétorique à l'analyse des publics », Réseaux, "Figures du public », vol. XXII, 126, Hermès Publications, 2004, p. 113-141).

35. Stuart Hall, Identités et cultures. Politique des Cultural Studies, Éditions Amsterdam, 2007.

36. Robert E. Goodin, "Democratic Deliberation Within”, Philosophy \& Public Affairs, 29(1), 2000, p. 81-109. 
l'espace public, qui légitime en retour le financement public des arts au nom des valeurs démocratiques. Nous faisons en somme le chemin inverse pour voir comment ce jeu des points de vue dans le cinéma et l'audiovisuel est lui-même une forme de délibération.

Dès lors, les hypothèses sur le sens qui traversent le spectateur le conduisent à opter pour une interprétation plutôt qu'une autre en fonction des points de vue alternatifs possibles qui se présentent à lui au cours de la lecture, en fonction du contexte, de ses ressources propres (de son histoire plus ou moins personnelle de spectateur, de son expérience du monde social) et des points de vue collectifs déjà disponibles quant aux formes ou aux enjeux identiques (ou similaires) à ceux avec lesquels il est aux prises au moment de la lecture, ou dans un moment de re-lecture ultérieure (par exemple au cours d'une discussion qui lui fait réviser son point de vue premier). Précisons, si cela est nécessaire, qu'il ne s'agit pas de savoir ce qui se passe dans la tête du spectateur (par exemple quel est le degré de conscience d'un spectateur lorsqu'il « choisit » une interprétation plutôt qu'une autre), mais de faire des hypothèses quant aux parcours interprétatifs possibles en confrontant des objets audiovisuels avec leurs réceptions attestées (et formulées publiquement ${ }^{37}$ ). Plus précisément, il s'agit d'observer les formes ${ }^{38}$ visuelles et sonores depuis les lectures spectatorielles telles qu'elles se manifestent à travers des interprétations en conflit. C'est donc aussi, en ce sens, une démarche qui est exactement l'inverse de celle du lector in fabula d'Umberto Eco ${ }^{39}$.

\section{Les lieux de la délibération}

Pour étudier ces interprétations, commençons d'abord par situer les différents niveaux auxquels on peut saisir le débat sur les objets audiovisuels (mais sans doute aussi culturels et médiatiques en général). Tout d'abord, qu'il s'agisse de délibération rhétorique (qui porte sur les enjeux à travers les objets) ou d'une délibération qui porte sur les formes poétiques prises par ces objets eux-mêmes, il faut 1 . envisager le film ou le document audiovisuel comme un débat sous forme d'images et de sons (ce n'est pas seulement une représentation, un monde, un récit...), réciproquement, 2 . considérer également que c'est à travers cette mise en forme (qu'on l'appelle " dispositif », « jeu formel », " agencement », etc.) que l'on peut penser le rapport du document audiovisuel à son contexte, mais aussi 3. partir de l'idée que cette mise en forme est elle-même construite par le spectateur en fonction de la façon dont il discute avec le document. 4. La façon dont il discute avec le document est elle-même prise dans une sphère publique de débat (un « espace public ») dans un lieu et un moment donnés. Enfin, conformément à

37. Ceci désigne exactement la limite de l'enquête mais aussi son objectif, il ne s'agit pas de se prononcer sur la psyché du spectateur mais d'observer la vie publique des signes et de leurs interprétations, en sachant que tout spectateur a une histoire de sa "spectature ", au sein de laquelle les dimensions psychiques jouent un rôle fondamental et peuvent même affleurer dans l'expression publique d'un jugement. On peut faire une place à cette dimension sans en faire l'objet de l'enquête.

38. Nous adoptons une définition large de «forme» (audiovisuelle) : toute organisation stabilisée de traits sonores et/ou visuels. Il importe en effet, comme nous le verrons plus loin, de ne pas prédéterminer ce que le spectateur va considérer comme « forme ».

39. Umberto Eco, Lector in fabula. Ou la coopération interprétative dans les textes narratifs, Grasset, 1985. 
ce que nous avons souligné tout à l'heure, 5 . cette sphère publique de débat n'est pas homogène et égalitaire, et on peut plutôt la considérer comme une sphère publique des débats, si l'on prend en considération l'histoire de cet espace public, sa connexion avec d'autres espaces et cultures, les stratégies des acteurs de cet espace, les effets d'agenda, les luttes de légitimité qui font que certains débats peuvent avoir lieu, d'autres non, ou sont peu audibles, etc.

Quelques exemples pour illustrer ces cinq lieux du débat (ou ces cinq manières de dire ou cristalliser le caractère " discutable » d'un film ou d'un document audiovisuel) : 1. Une femme est une femme propose un point de vue sur l'émancipation féminine à travers son intrigue et sa mise en scène ; les choix de scénographie, le tour de parole et le rôle des journalistes dans un débat télévisé de second tour doivent viser l'impartialité. 2. Le caractère patriarcal des films classiques s'exprime dans les rapports de sujets regardants à objets regardés ; les dispositifs des talk-shows expriment un imaginaire contemporain de la démocratie, variable selon les cultures ${ }^{40}$. 3. Un spectateur de 1963, qui identifiera sans difficulté dans Muriel, de Resnais, le personnage qui représente "l'Algérie française »(Alphonse), s'appuiera sur le système d'opposition des personnages pour appréhender le point de vue du film sur les " événements d'Algérie »; un téléspectateur ouvrier anglais syndiqué appréhendera différemment la nécessité de baisser les salaires défendue dans un débat à la télévision ${ }^{41}$, par rapport, disons, à un petit commerçant londonien. 4. Si l'on imagine un(e) spectateur/-trice féministe des années 1970 d'Une femme est une femme, le « désir d'enfant » d'Angela est certainement situé par ce(tte) spectateur/-trice par rapport à la position de certains courants féministes qui visaient à libérer les femmes de la maternité ; la critique de la " télé-poubelle » (trash-TV) s'appuiera davantage aujourd'hui sur les enjeux éthiques de la " télé-réalité » que sur la présence à l'antenne de feuilletons et séries télévisés américains qui sont aujourd'hui valorisés. 5. Lorsque Serge Daney s'intéresse à Citizen Kane à la fin des années 1980, il évoque «la critique des médias » d'Orson Welles, utilisant un paradigme argumentatif en plein essor $^{42}$; l'accès à la sphère publique des premiers « écologistes » était difficile dans les années 1950-1960, là où, aujourd'hui, les émissions de télévision tenant un propos écologiste sont nombreuses et légitimes, une perspective écologiste est donc beaucoup plus aisée à mettre en œuvre et à défendre publiquement aujourd'hui. Et ainsi de suite.

Cette première échelle du débat, depuis l'organisation historique des espaces publics jusqu'à l'appréhension sémiotique des formes, permet donc de situer en son sein la dynamique délibérative de lecture comme un processus de construction

40. Patrick Charaudeau et Rodolphe Ghiglione (éd.), Paroles en images. Images de paroles. Trois talkshows européens, Didier Érudition, 1999.

41. Par opposition à la lecture "préférentielle » (favorable à l'idéologie dominante du Centre), on peut trouver des " lectures oppositionnelles ", comme « le cas d'un spectateur qui assiste à un débat sur la nécessité de limiter les salaires mais qui "lit" toute mention de l"'intérêt national” comme "intérêt de classe" " (voir Stuart Hall, Encoding/Decoding in Television discourse, Archives du Center for Cultural Studies, Stencilled occasional papers, Media series, SP 7, Université Birmingham, 1973, p. 138).

42. Serge Daney, Devant la recrudescence des vols de sacs à main, cinéma, télévision, information : 19881991, Aléas, 1991. 
progressive des points de vue du document audiovisuel corrélativement à l'activation ou à la réactivation des différentes positions culturelles, sociales et politiques que peut adopter le spectateur, dans un triple rapport à son expérience personnelle des images et des sons, aux positions collectives (en général publiques, mais pas toujours) susceptibles de s'exprimer par rapport au même objet et enfin à l'évolution du document audiovisuel lui-même. Le caractère temporalisé de l'expérience audiovisuelle est en effet une caractéristique particulièrement importante du film ou du programme de télévision, favorisant la bifurcation, la transformation ou la révision d'une ou plusieurs interprétations de départ, et nombreux sont les objets audiovisuels qui s'appuient en réalité sur l'évolution du jugement spectatoriel, ou en jouent (bien souvent, on n'y voit pourtant qu'un geste démiurgique - un coup de théâtre ou un coup de force - de l'" auteur »).

Prenons comme exemple le film Reprise (Hervé Le Roux, 1997), parfois qualifié de « polyphonique » et dont nous voudrions montrer qu'il est un film qui, certes, propose plusieurs points de vue sur Mai 68, mais qui, surtout, ne peut tout simplement pas fonctionner si l'on ne présuppose pas cette activité sémiotique délibérative que nous cherchons à décrire. Deux séquences qui se font écho dans le film, à une demi-heure d'intervalle, peuvent nous aider à le voir. Vers la fin du petit film tourné en juin 1968 par des étudiants de l'Idhec, qui est le point de départ de celui de Le Roux ${ }^{43}$, on voit des ouvriers commencer à rentrer dans l'usine Wonder à l'invitation du chef du personnel. Ce fait de rentrer dans l'usine est perçu différemment par deux protagonistes de l'époque auxquels on projette ce film de 68, l'un ancien conseiller municipal communiste de Saint-Ouen, favorable à l'époque (comme on le voit dans l'archive), comme aujourd'hui encore, au fait de " savoir arrêter une grève », l'autre, une ancienne militante gauchiste, animatrice de la grève à l'intérieur de Wonder. Le premier revit la scène avec un certain soulagement et esquisse un sourire : "Ils rentrent », l'autre, attristée, prononce les mêmes mots mais sur un tout autre ton, en poussant un soupir, puis elle prend une cigarette dans son paquet, avant de reparler. Comme auparavant dans Reprise, l'archive de 68 s'enrichit donc de nouveaux points de vue sur la scène : le conseiller municipal, par exemple, nous apprend qu'il avait fait auparavant de la prison en Algérie pour insoumission, ce qui, bien sûr, donne une plus grande complexité à son attitude devant Wonder, tandis que la militante gauchiste explique bien les rapports entre grévistes de l'intérieur et organisations syndicales et politiques à Saint-Ouen. On voit aussi comment chacun des protagonistes demeure en quelque sorte fidèle à lui-même. Le spectateur est incité à emprunter alternativement le regard de chacun d'eux sur la scène (à l'époque comme aujourd'hui), ce qui peut enrichir ou faire évoluer son propre point de vue à mesure que l'on rencontre des protagonistes de l'époque et que l'on revoit cette scène inaugurale - c'est tout le projet du film -, mais aussi à juger et comparer les points de vue entre eux, y compris le sien propre (selon que l'on a connu ou participé à Mai 68, ou non ; ou en

43. Il s'agit de retrouver la jeune femme qui, dans ce film, s'oppose fortement, avec rage et pleurs, à la reprise du travail, conduisant à revoir tous les protagonistes de cette scène, ce qui amène à restituer et revisiter à la fois ce moment-clé de 68, mais aussi la vie dans une usine de l'époque, l'évolution de la condition ouvrière et la mémoire de Mai 68 depuis, entre autres. 
fonction d'une lecture gender telle qu'on pourrait la proposer aujourd'hui : femmes révoltées contre apparatchiks masculins, ou postcoloniale : absence à l'écran des ouvriers algériens dont il est pourtant fait mention par les protagonistes, etc.).

Ce travail du spectateur n'est pas propre à Reprise, ni même au documentaire en général (pensons en particulier aux fictions qui comportent un enjeu social ou politique - soit presque toutes... -, aux films historiques, aux dystopies de la science-fiction, etc.), et tout film peut faire l'objet d'un investissement de ce type de la part d'un spectateur, mais, ici, il s'explicite. Ce qui produit le sentiment, souvent noté, d'une " archive vivante ", c'est-à-dire que, sans en passer par une distanciation brechtienne (bien au contraire, pourrait-on dire), le spectateur, pour participer et prendre du plaisir et de l'intérêt à suivre le film, doit changer son rapport à l'archive filmique relativement aux formes traditionnelles du « documentaire d'archives » et de l'enquête par témoignages (entretiens), qui visent, elles, en général, à défendre un point de vue donné, en s'appuyant soit sur des témoins, soit sur des archives vues une fois, à l'appui de tel moment du propos. La délibération spectatorielle porte donc à la fois sur les enjeux (Mai 68, la mémoire de 68, l'histoire de la condition ouvrière, le sens des luttes, le renouveau militant après les grandes grèves de 1995, etc.) et sur les conventions du film documentaire et du film politique ${ }^{44}$. Outre l'explicitation du travail spectatoriel,

44. Quelques exemples de réactions qui illustrent ce travail interprétatif (je souligne) : « [le film ne laisse pas indemne] pour preuve, les réactions des spectateurs invités à participer à un débat. "On est nombreux aujourd'hui à nous demander quoi faire; il me semble que c'est le sujet au centre de ce film”, lance une première intervenante. "On a l'impression que toutes les questions posées il y a une trentaine d'années sont restées en suspens", poursuit une voix dans la pénombre. [...] Plusieurs doigts se lèvent. Surtout des femmes, qui travaillent à la chaîne. Et pour lesquelles “ça n'a pas beaucoup évolué [depuis 1968], c'est encore la galère". "ÇA VA REVENIR" "Le film n’est pas sur 1968, ce n'est pas un film de souvenirs", poursuit un jeune homme. Pour Jean-Marc, quarante ans, dont vingt-quatre passés à la chaîne, "ce film est le reflet de la société actuelle ; ce que disent les ouvriers, c'est ce qu'on ressent ; il y a une espèce d'amertume, de désillusion". "Ça va revenir", dit une femme à voix basse. "Il faudrait faire un film sur les gens qui vont voir le film", susurre une autre. [...] "Ce n'est pas un film sur le souvenir, mais sur la mémoire, estime Armand. Le souvenir, c'est mort, alors que la mémoire, c'est quelque chose qui est présent." " (Marie-Pierre Subtil, article du Monde sur les débats organisés autour du film, 2 mai 1997) ; "Reprise est une saga où les personnages échappent à leur étiquette pour devenir des blocs de vie qui vous sautent à la tête. Vingt-sept ans après le brûlot des étudiants de l'Idhec, Hervé Le Roux a écouté tous ses "héros “ en leur donnant le temps de s'exprimer, et c'est une découverte! Au temps de la parole coupée, manipulée, ce film est une leçon, un témoignage unique sur la façon de restituer la vérité des êtres. Pas de jugement, pas de prise de pouvoir par le réalisateur ; tous les protagonistes sont filmés dans la lumière chaude de l'été. C'est comme si, brusquement, ils s'échappaient de la photo fixe. Ils se mettent en mouvement, ont droit à la complexité. » (Catherine Humblot, Le Monde, 18 mai 1997) ; «Un film, une fille, une grève : Reprise est bel et bien un film sur le cinéma, un film romantique et un film politique. Un traité sur le cinéma comme enregistreur de traces et machine à penser, un ample portrait de femme motorisé par le fantasme, une plongée dans la mémoire ouvrière. En revoyant le film aujourd'hui, on est convaincu qu'Hervé Le Roux est heureux de ne pas avoir retrouvé la fille au bout de sa longue (en)quête. » (Serge Kaganski, à l'occasion de la sortie du DVD, Les Inrockuptibles, $1^{\mathrm{er}}$ janvier 2004) ; "Reprise, c'est ça. Un aller-retour passé présent. Ouvriers-retraités. Visages d'avant, Regards d'aujourd'hui. Ceux de chez Wonder s'appellent et se rappellent. Les petits chefs, les premières ouvrières, les contremaitres et les ouvrières tout court. [...] Trois heures comme ça. Dans le capitalisme à la papa des années 60 finissantes. Puis dans celui, néo-libéral, à la Tapie. Où tout finit toujours pareil, où tout finit comme il se doit : en solde de tout compte. À la fin, le chef du personnel, il dit que "les gens de chez Wonder doivent reprendre le travail”, il précise : “...calmement”. Et elle, Jocelyne, sublime/sublimée, hors-cadre, qui dit juste ça, le plus beau “c'est ça !” jamais prononcé. “C’est ça !”, comme basta. [...] Et 
c'est la mise en relation entre ces deux formes de délibération qui explique donc certainement l'investissement particulier des spectateurs et la réussite critique et publique du film.

\section{Les niveaux de la délibération}

La deuxième échelle de la délibération, qui croise la première, concerne justement le niveau de mise en jeu du simulacre audiovisuel : comment le débat se manifeste-t-il quant au jeu formel ? En reprenant ce que nous avons vu jusqu'ici et en le prolongeant, nous pouvons distinguer trois saisies délibératives. La première considère que les enjeux sociopolitiques font l'objet d'un traitement audiovisuel, c'est donc un enjeu que l'on vise à travers le jeu des formes (le dispositif de Le Roux permet de multiplier les points de vue sur 68, confrontés au(x) point(s) de vue du spectateur). C'est le niveau rhétorique que nous avons déjà présenté. La deuxième saisie interroge les conventions audiovisuelles : le documentaire de Le Roux peut être vu comme un simple "reportage ", ou, à l'inverse, comme le contraire d' " une investigation documentaire, reconstituant sur un mode didactique les conditions de travail dans les anciennes usines Wonder [...] s'ordonnant selon l'équilibre traditionnel : questions-réponses, entretiens-documents d'archives, exposer les faits en somme, avec une apparence d'objectivité qui en appellerait à la conscience (et à la patience) du spectateur [...] [car] le projet [de Reprise] est d'une autre nature [...]» ${ }^{45}$. Il peut être vu comme un film romantique attaché à une figure féminine ${ }^{46}$, ou au contraire comme un film qui illustre une condition générale (" elle ou une autre [...] des ouvrières qui ne voulaient pas rentrer, il y en avait dans tout le pays ») ${ }^{47}$, comme un film hybride (" polar social (qui a tué la classe ouvrière ?), [c'est un] livre d'histoires françaises et d'Histoire de France, et aussi, et surtout, [une] romance amoureuse ${ }^{48}$, repris, entre autres, sur la pochette du DVD en 2004), comme une réponse du cinéma à la télévision (certains spectateurs comparent le film aux Dossiers de l'écran en regrettant que la télévision ne propose plus ce genre de discussion), etc. Il s'agit de situer la discussion avec les images et les sons par rapport aux habitudes spectatorielles telles qu'elles sont sédimentées par les expériences personnelles ou collectives, les canons officiels ou parallèles, les grandes catégories génériques, les types de films ou de médias. Un exemple particulièrement parlant nous est apparu en travaillant sur Les Bureaux de Dieu (2008), documentaire de Claire Simon ayant recours à des - toi, tu deviens quoi? Mollesse de tes "c'est ça”. Faiblesse de tes faiblesses. Wonder ? La vie ne s'use que si l'on ne s'en sert pas. » (David Dufresne, 24 avril 2004 - www.davduf.net).

45. Noël Herpe, Positif, 434, 1997.

46. " Mais que ce soit à New York ou à Lugron (Landes), ce sont souvent les mêmes interrogations qui reviennent. Et d'abord la question : "L’avez-vous retrouvée ?" "Pas jusqu’à ce soir, à moins qu'elle n'habite New York." Petits rires nerveux, regards suspicieux vers la quinquagénaire la plus proche, puis gros soupirs de déception dans la salle ; désir clamé haut et fort de rencontrer cette femme qui crie ("J’aurais moi aussi aimé la rencontrer, dit un spectateur à Le Roux, désolé pour vous") ", Frédéric Bonnaud, à propos d'une projection à New York du film, Les Inrockuptibles, 8 avril 1998.

47. Réactions recueillies par Hervé Le Roux lui-même et retranscrite dans le livre publié en 1998 qui prolonge le film (Hervé Le Roux, Reprise, Calmann-Lévy, 1998, p. 93).

48. Gérard Lefort, Libération, 26 mars 1997. 
actrices connues pour redire des paroles entendues au sein de plusieurs antennes du planning familial, un spectateur cherchant à situer progressivement le film :

Ce qui peut ressembler à un film à sketchs, puisque s'enchaînent différents entretiens sans qu'il n'y ait de suite narrative pour l'un d'entre eux, est un quasi-reportage totalement passionnant. [...] Bien que les problèmes évoqués parleront davantage aux spectatrices, chaque scène est captivante et donne vraiment envie de connaître le nœud du problème. Je n'ai pas vu passer les deux heures de film et je ne sais plus si ce sont les performances des actrices [...] ou la puissance de chaque situation qui donne le plus d'intérêt à la narration [...] Côté « scénario » il y a un crescendo qui s'étend des problèmes de femmes jeunes vers ceux des plus mûres. (Shazam12, Allociné.fr, 9 décembre 2008)

On remarque qu'une première façon de donner une forme au film est écartée (« film à sketchs ») au profit d'une approximation donnée comme telle (« quasi-reportage »), puis, quittant le recours au genre pour identifier les formes, l'internaute reconstitue progressivement la façon dont les «problèmes " (le terme intervient quatre fois) évoqués prennent sens autour de quatre enjeux formels (scène, situation, narration et scénario). Ces " problèmes " produisent du même mouvement les formes du film, la position du spectateur et enfin l'enjeu du film, dans une progression logique de la scène (qui n'est pas un sketch) au film dans son entier (crescendo du scénario ${ }^{49}$. On voit bien ici que c'est en fixant progressivement le type de film auquel il a affaire que le spectateur parvient à situer l'enjeu. Notons également que, tout autant que la délibération sur les enjeux, la délibération sur les conventions formelles engage la position du spectateur par rapport aux autres spectateurs réels ou imaginés : le rédacteur évoque ici la différence entre spectateurs et spectatrices. Dans un tout autre genre, un internaute évoque la différence entre les amateurs de Quentin Tarantino et les amateurs de la série CSI (Les Experts) à propos d'un double épisode de CSI, " Grave Danger », réalisé par le cinéaste :

Je suis en général assez partagé vis-à-vis des films de Quentin Tarantino [...] on pourrait m'objecter qu'il est le Godard de sa génération [...] [en tout cas] la première partie de Grave Danger ne déçoit pas, que vous soyez ou pas un fan de Tarantino ou de CSI. [...] mon avis est que Tarantino réussit à combiner harmonieusement les habituels éléments de thriller de CSI avec sa propre marque de fabrique. (UlrikSander, imdb.com, 26 septembre 2006, ma traduction)

Les genres sont bien compris, dans un cas comme dans l'autre, non seulement comme des conventions fixées par l'histoire ou l'industrie, mais comme des relations (liées au goût, au fait d'être concerné, etc.) entre spectateurs et films, relations dont on discute, et c'est en se glissant dans cette discussion, en la réactivant, que le spectateur parvient à situer son propre rapport au film, aux conventions et à la discussion sociale sur films et conventions.

49. Pour une analyse plus détaillée de la réception délibérative des Bureaux de Dieu, je me permets de renvoyer à Guillaume Soulez, "Les agrégats délibératifs : et s'il n'y avait pas de "communauté" d'interprétation ? La réception délibérative des Bureaux de Dieu par les internautes ", in Marta Boni, Adrienne Boutang, Barbara Laborde et Lucie Merijeau (éd.), « Networking images, approches interdisciplinaires des images en réseau », Théorème, 17, 2013, p. 119-129. 
La troisième saisie possible au sein de cette deuxième échelle de la délibération concerne le simulacre lui-même. Plus rare, cette délibération porte sur le rapport au monde à travers images et sons, en quelque sorte en deçà de la représentation. Hervé Le Roux rapporte le cas de ces spectateurs qui pendant des débats après la projection de Reprise considéraient que même la scène de juin 1968 était jouée par des acteurs ${ }^{50}$, là où, tout au contraire, plusieurs spectateurs et critiques voient dans ce plan-séquence la quintessence du cinéma-vérité ${ }^{51}$. La crise ouverte par le caractère incroyable des images du 11 septembre 2001 offre un exemple paradigmatique de cette dimension. Pour préciser ce qui est en jeu, on peut s'appuyer sur le travail de Jean-Marie Schaeffer dans L'Image précaire autour de ce qu'il appelle la «thèse d'existence » liée à l'archè (l'origine) photographique : nous « savons » que la photographie (ou le cinéma, la télévision) repose(nt) sur des empreintes du réel (photochimique, par balayage électronique, etc.), nous faisons donc l'hypothèse que quelque chose est capturé par l'image ( "ça a été là », comme le notait Barthes), mais s'ouvre alors tout l'empan des possibles liés à la désignation, au découpage du réel, à la prise en compte du hors-champ, du contexte, etc ${ }^{52}$. Nous ne nous en rendons pas compte, couramment, aiguillés par nos habitudes de lecture conventionnelles et les légendes ou les commentaires qui les réactivent, mais il arrive que ce ne soit pas le cas et que la discussion porte précisément sur la question de savoir ce à quoi nous avons affaire exactement.

Un passage d'Une femme est une fermme, peu après celui que nous avons évoqué plus haut, relève également de cette interrogation : Godard a visiblement conservé au montage une erreur, voire un lapsus, d'Anna Karina qui, devant faire dire à son personnage, Angela, qu' « elle trouve con les femmes modernes qui essaient d'imiter les hommes ", commence par dire, à la place : "d'éliminer ». Puis elle commente d'un air triste et d'une façon qui s'adresse à la caméra : "Non, ça ne va pas ». Le débat peut s'engager (et il ne manque pas de s'engager dès qu'on pointe le passage) sur le sens de cette séquence, la raison de la conserver (alors que la bonne prise est collée à sa suite), sa fonction argumentative (critique de l'aliénation d'Angela, mise au jour ou au contraire renforcement de la domination masculine sur l'actrice, ou enjeu « purement » esthétique), etc. On voit en tout cas que ce troisième niveau de délibération se distingue bien de l'habituel brechtisme godardien dans le même film, lorsque celui-ci fait, par exemple, saluer les acteurs " en représentation », avant que ne reprenne la «comédie » entre eux, manière de

50. Frédéric Bonnaud, art. cit. Voir aussi, explicitant bien ce passage de la délibération des conventions à la délibération sur le simulacre lui-même, cette réflexion finale de Gérard Lefort à la sortie du film : «Cette métamorphose permanente du regard induit que pas une seule des 192 minutes du film n'ennuie. Mais Reprise nous regarde à son tour et fascine par sa manière tendre mais insistante de débusquer quelques questions de fond [...] qu'est-ce qu'une image ? qu'est-ce qu'elle nous dévoile et nous cache dans le même mouvement ? comment la lit-on ? Et même, par un bel effet de boomerang autocritique : de quelle idéologie relève aujourd'hui notre confiance aveugle ou notre méfiance éclairée pour tout ce qui se pare de la caution "documentaire"? (Gérard Lefort, art. cit.).

51. Un exemple parmi cent autres : «Un court film documentaire (un seul plan séquence) qui a toutes les qualités d'une excellente photo de reportage, et avec le même caractère miraculeux. Être là au bon moment, avoir la présence d'esprit, savoir capter... Toujours est-il que c'est un concentré dramatique du mouvement de 68 finissant coté ouvrier. En tant que tel c'est devenu une icône du cinéma vérité. ", benoitparis, Allociné.fr, 22 février 2011.

52. Jean-Marie Schaeffer, L'Image précaire, Seuil, 1987.

communication \& langages $-n^{\circ} 176$ - Juin 2013 
fragiliser l'enveloppe poétique pour mieux faire apparaître le caractère artificiel de la fiction, mais aussi son charme (niveau 2), et, du coup, faire émerger le caractère typique de ce couple et le débat social sur l'émancipation féminine, c'est-à-dire favoriser in fine la délibération rhétorique (niveau 1), si le spectateur ne l'avait pas engagée d'emblée.

Une description satisfaisante de la délibération spectatorielle suppose donc le croisement de ces deux échelles - des formes audiovisuelles aux espaces publics et des enjeux au simulacre - ou, de façon simplifiée, échelle du débat (lieux de la délibération) et échelle des enjeux (niveaux de la délibération) - permettant de prendre la juste mesure de tel ou tel débat, et de tel ou tel processus sémiotique de production du sens. Dire que Les Bureaux de Dieu "n'a rien de cinématographique » mais qu'il pourrait être diffusé « [au] collège ou [dans] les lycées ${ }^{53}$, c'est défendre une certaine conception du cinéma. . . tout en considérant que le " message » éducatif que le film contient serait utile à diffuser au sein de notre espace social et politique. Ceci s'analyse comme une délibération relevant du niveau conventionnel (2) des enjeux et permettant de situer le débat par rapport à l'état actuel de notre espace public - lieu (4) - du film comme débat. Considérer que Reprise est une « leçon de cinéma » sur l'archive, c'est situer le débat au niveau formel lui-même - lieu (1) - tout en permettant une discussion sur les usages et les normes du documentaire (ou du reportage) d'archive traditionnel - ce qui relève là aussi du niveau (2) des enjeux. Dire en 1961, à propos d'Une femme est une femme, que Godard s'amuse avec un problème sérieux, voire le méprise ${ }^{54}$, peut s'analyser comme une mise en débat qui relève de la façon dont le film est vu comme proposant un certain point de vue sur le contexte contemporain (lieu 3), mais permet aussi d'accéder par la comparaison avec d'autres réceptions possibles du film à la position des spectateurs qui proposent cette lecture critique (lieu 2 des débats), ce qui se fait ici en soulignant le caractère inapproprié du jeu formel pour traiter un enjeu social (niveau 1 des enjeux).

\section{DÉLIBÉRATION, RÉCEPTION ET « MISE EN FORME(S) »}

Ce type de questionnement et cette méthode des échelles permettent ainsi une certaine cartographie de la réception : si l'interprétation d'un film peut paraître en apparence " infinie » en fonction de la diversité des individus, le nombre de débats et la forme qu'ils prennent - les interprétations en conflit - ne sont, eux, pas du tout infinis. Ils se structurent en général autour de trois ou quatre nœuds principaux, correspondant à l'état social et politique de l'espace culturel considéré. Ce qui nous donne, bien souvent, non seulement une photographie de cet espace sous une lumière donnée, mais aussi une idée des représentations et des attentes des spectateurs par rapport au cinéma et aux médias (audiovisuels). Il

53. "Rien de cinématographique dans ce docu-fiction. [...] intéressant dans l'idée et dans le fond on s'aperçoit vite que ce film n'est pas indispensable car c'est beaucoup trop survolé [...] il s'agit avant tout d'une accumulation de témoignages. [.. . A À la rigueur c'est un film pédagogique pour le collège et les lycées, pour une diffusion sur F3 et/ou Arte mais ce film n'a absolument rien à voir avec le cinéma » - selenie, Allociné.fr, 4 mars 2010.

54. Dans Télérama et dans Le Figaro en 1961, voir Geneviève Sellier, La Nouvelle Vague : un cinéma au masculin singulier, CNRS Éditions, 2005, p. 137-138 et p. 140. 
importe bien sûr de situer ces délibérations dans leur espace propre d'apparition : débattre d'un film ou d'un programme de télévision après l'avoir vu en famille ou avec des amis suppose de comprendre comment l'espace public peut traverser (et rendre conflictuel au sens large) l'espace familial ou amical, ce qui sera différent de situations où le débat public fait partie des attendus (par exemple les forums de discussion sur le cinéma, sur la télévision ou sur tout autre sujet qui implique des films, des programmes, etc. ; ou les « ciné-débats », qui prennent aujourd’hui la relève des ciné-clubs). De même, l'expertise professionnelle conditionne la prise de parole et les formes d'interprétations d'un critique de cinéma ou de télévision, enfin, certaines œuvres sont des formes de délibérations d'autres œuvres (pensons, par exemple, aux cartoons de Tex Avery, par exemple TV of Tomorrow, 1953). Nous n'avons pas la place ici de développer cette part du prisme de l'espace d'apparition considéré, mais il entre bien sûr en interaction avec les lieux et les niveaux de délibération et doit faire partie de l'analyse de tel ou tel cas. En particulier, étudiant la délibération d'un film ou d'un document audiovisuel, nous traversons différents types de discours, de la réaction ordinaire à la critique professionnelle, non pas pour tenter de les répartir selon des ensembles qui correspondraient aux normes du goût et au degré de maîtrise des codes (et de la formulation du jugement), mais pour, en quelque sorte, "les retourner comme un gant ": non pas pour voir ce qu'ils veulent dire, mais pour observer en sens inverse ce qui, en eux, malgré ou grâce à ce prisme, ressemble ou diffère d'autres arguments, d'autres constructions du sens du même film ou du même document audiovisuel, et ce, avec son lot de surprises.

\section{Une double mise en relief}

On opère donc une double mise en relief : de la réception en tant que délibération plutôt que reconnaissance (comment les spectateurs s'emparent du film ou du document audiovisuel pour discuter avec lui et avec leur propre contexte social, politique, esthétique, etc.), mais aussi du dispositif lui-même de ce film ou de ce document audiovisuel, dans la mesure où il s'agit de faire ressortir certains traits audiovisuels que cette mise en débat va souligner et qui seraient demeurés dans l'ombre sinon. Par-là, en observant comment les formes sont mises en débat, on peut éviter certaines impasses, par exemple autour de la question du " réalisme » d'un film ou d'une série télévisée. Bien souvent cette catégorie poétique, fondamentalement mimétique car fondée sur la ressemblance, nous masque des enjeux délibératifs : quand on dit d'un film qu'il est " réaliste » parce qu'il aborde sans tabou certaines questions, on se prononce, en fait, non sur le processus mimétique mais sur la prise de position du film dans un espace public, ce que l'analyse délibérative permet de mettre au jour. En ce sens, notre démarche s'apparente par certains aspects à la microstoria de Carlo Ginzburg, qui vise en particulier à retrouver le contexte dans le cas, suivant ce que l'historien a appelé le " paradigme indiciaire », privilégiant, comme le note François Rastier, 
« les parcours d'interprétation sur la typologie des signes » ${ }^{55}$, en procédant par abduction (plutôt que par déduction ou induction). Notre démarche abductive s'appuie ainsi principalement, comme on l'a vu, sur la décantation que permet la comparaison des lectures du même objet. Ce faisant, le chercheur s'approche par sa méthode de la logique inférentielle du spectateur lui-même qui fait, à mesure du film ou du document audiovisuel, des séries d'hypothèses sur les formes et leurs lectures possibles, qui confirment ou infirment son positionnement, son jugement en cours.

Le travail délibératif, que Reprise, notamment, rend visible, consiste donc à faire comparaître la pluralité des sens possibles pour sélectionner - souvent provisoirement - une hypothèse qui est la forme et le moyen pour un spectateur d'accepter ou d'assumer une position, c'est-à-dire de situer cette position parmi d'autres possibles chez d'autres spectateurs. En ce sens, la discussion publique n'est pas une sorte de second moment de la réception, mais elle traverse le spectateur au moment de l'interprétation. Il s'agit donc ici de pouvoir étudier, à côté de la capacité des spectateurs à reconnaître des codes, d'une part, la façon dont les bifurcations de l'interprétation se produisent, d'autre part, les liens entre ces bifurcations et l'ancrage social des hypothèses de lecture.

Inversement, ce que nous appelons " délibération des images et des sons » ne concerne pas les débats qui portent sur autre chose que ces images et sons, et qui, de fait, souvent ne les évoquent pas. Nous avons rencontré en étudiant la " réception ", de 2008 à 2010, des Bureaux de Dieu dans les forums d'Allociné.fr, et sur quelques autres sites, six débats principaux. Les quatre premiers vont du plus orienté vers une discussion sur les conventions poétiques au plus orienté vers une discussion sur les enjeux sociopolitiques : 1. un débat sur le caractère documentaire ou non du film, 2 . un débat sur les choix documentaires eux-mêmes (modes d'enquête, choix de filmage. . .), 3. un débat sur la restitution du « réel » (le planning familial fonctionne-t-il vraiment comme le montre le film), 4. un débat sur les sujets abordés (contraception, avortement, questions communautaires, enjeux de la liberté sexuelle) et le discours que le film tient sur ces sujets à travers ses choix de casting, de mise en scène... Mais deux autres débats ont également eu lieu sur ces forums : l'un qui porte sur la définition en général du cinéma et du documentaire, l'autre qui porte sur les "contenus » eux-mêmes (IVG, contraception, etc.), certains internautes n'ayant visiblement pas vu le film : ce sont deux discussions qui se développent sans lien avec les formes spécifiques du film ${ }^{56}$. Ainsi, on ne dira pas qu'il y a une limite à l'interprétation car rien ne permet véritablement de trouver une limite textuelle qui distinguerait l'interprétation de la simple « utilisation », pour reprendre la célèbre distinction d'Eco ${ }^{57}$, on va y revenir,

55. François Rastier, «Indices et parcours interprétatifs », in Denis Thouard (dir.), L'interprétation des indices. Enquête sur le paradigme indiciaire avec Carlo Ginzburg, Septentrion, 2007, p. 123. Ce n'est bien sûr pas un hasard si, en s'appuyant sur la tradition argumentative de la Rhétorique, Ginzburg a ferraillé du même mouvement contre les tentatives de réduire le récit de l'historien à une " fiction », voir Carlo Ginzburg, Rapports de force. Histoire, Rhétorique, Preuve, Gallimard, 2003.

56. Guillaume Soulez, art. cit., 2013.

57. Umberto Eco, Les limites de l'interprétation, Le livre de poche, 1994. 
en revanche, il y a une limite à la délibération des images qui est celle justement qui consiste à débattre de questions sans établir dans l'interprétation de rapports entre ces débats et les formes audiovisuelles elles-mêmes.

\section{Mise en forme(s)}

De ce point de vue, prise littéralement, la notion de « réception » se trouve presque en contradiction avec l'hypothèse de la délibération, sauf à remonter à l'un de ses usages en histoire des idées quand il s'agissait, par exemple, de montrer comment la " réception » d'un auteur ou d'une pensée dans un autre contexte que celui d'origine produisait un nouvel auteur, une nouvelle pensée (un Montaigne anglais, un Shakespeare romantique, etc.). C'est une ruse étrange de l'histoire de la théorie que la notion de "réception ", qui convient bien à un modèle stimulus-réponse, ait servi à réhabiliter le rôle du lecteur. C'est pourquoi un pragmaticien comme Roger Odin utilise plutôt la notion de " production de sens ${ }^{58}$. Or, en observant la façon dont les spectateurs produisaient du sens à propos des programmes de télévision (enquête sur le courrier des spectateurs de Télérama en 2000 et enquête sur les courriels reçus à la Médiation de France Télévisions en 2004), nous avons fait l'hypothèse qu'ils ne se contentaient pas de produire du sens mais aussi des formes $^{59}$. Un spectateur choqué par la violence d'un accident lors d'un Paris/Dakar considérait les images comme un projectile, ce qu'on retrouvait ultérieurement d'une façon proche chez certains spectateurs du 11 septembre 2001. Certains spectateurs «traversent » l'écran pour être en relation avec un spectacle (musique, sport), d'autres (ou les mêmes, dans le même courrier, selon le parcours de leurs positions de lecture) s'arrêtent à la « surface » pour décrire tel effet de montage, tel cadrage qui leur semble leur tenir un discours auquel ils adhèrent ou qu'ils contestent : les mêmes plans rapprochés sur des handicapés lors du Téléthon peuvent donner lieu, par exemple, à des commentaires critiquant le «voyeurisme » de l'émission, tandis que d'autres spectateurs y voient favorablement une forme de levée de la censure visuelle sur le handicap. De même, il ne s'agit pas de dire que Reprise est " polyphonique » parce que nous voyons plusieurs points de vue s'exprimer, mais d'observer que c'est notre travail de spectateur qui fait varier l'archive elle-même au fur et à mesure du film, en fonction du débat dans lequel nous la situons, et qui nous fait noter tel détail ou tel plan, rapprocher le film d'un autre, etc. La «polyphonie» apparente du film n'est qu'une polyphonie représentée. En d'autres termes : la polyphonie est ici un support possible pour la délibération, mais la délibération ne se réduit pas à la polyphonie représentée par la diversité des points de vue des protagonistes, y compris le point de vue porté par le commentaire en voix-off. Ainsi, ce moment où nous évaluons et soupesons les significations tout en les fabriquant, parce qu'elles sont encore partiellement informulées, ce moment construit, du même mouvement, le " texte " que nous lisons et le "spectateur " que nous sommes.

58. Roger Odin, op. cit. 1990.

59. Guillaume Soulez, art. cit., 2004. 
Idée déjà présente dans sa réflexion sur la " figurativisation ${ }^{60}$, où un même film peut être construit différemment par les spectateurs selon le cadre et les consignes de lecture proposés (un même film peut être lu comme un documentaire sur la prolifération des molécules ou comme une œuvre expérimentale à base de pellicule grattée), Odin parle clairement aujourd'hui de " production du texte » par le spectateur à partir de vibrations (sonores ou visuelles) ${ }^{61}$. La difficulté avec la notion de texte est qu'elle suppose une cohésion que l'on confond bien souvent, dans la logique poétique d'engendrement du texte, avec la cohérence. Pour prendre un exemple simple, la "règle des trois unités " suppose trois dimensions qui vont faire la cohésion du texte (temps, lieu, action) au nom d'une norme du « vraisemblable » poétique qui assure la cohérence de la pièce de théâtre. La théorie poétique depuis Aristote superpose et articule en fait deux projets, s'interroger sur la mimésis (et l'engagement du spectateur dans cette mimésis) et comprendre les grands équilibres, les structures d'un artefact (pièce de théâtre, roman, film. . .) qui s'expliquent en fonction de règles de composition ${ }^{62}$. D'où les différentes théories morales et politiques (les normes de cohérence) qui ont été projetées sur la Poétique d'Aristote, sans que sa théorie de la composition ait été fondamentalement remise en cause, y compris chez les Formalistes russes. En particulier, ce qu'on peut appeler l'argument « toutiste » : les parties se rapportent au tout et le tout est plus important que la somme des parties. Or, la cohérence d'une composition est un effet de perspective, en particulier une projection de ceux qui «façonnent » sur l'objet façonné (sans parler du poids d'un modèle organiciste). Un spectateur peut, en fonction de sa délibération et de ses changements de position, relier deux ou plusieurs aspects ${ }^{63}$ visuels ou sonores, parfois éloignés, pour considérer qu'il a affaire à une forme particulière (on l'a vu avec le spectateur qui effectue un parcours de lecture depuis le « sketch » jusqu'au "scénario »). Il peut donc tout à fait produire une autre cohésion formelle, qui paraîtra « incohérente » à celui qui a façonné, mais qui n'en est pas moins une manière de tenir ensemble différents aspects sonores et visuels.

Les linguistes ont souvent constaté qu'un énoncé qui contient pourtant des marques de cohésion (par exemple une phrase qui commence par une majuscule et se termine par un point) peut être incohérent ${ }^{64}$. Ce que cherche à faire précisément la poésie sans ponctuation, c'est d'inciter, à partir d'une faille dans

60. Roger Odin, De la fiction, De Boeck, 2000, p. 18.

61. Roger Odin, Les Espaces de communication, Presses universitaires de Grenoble, 2011, pp. 18-21.

62. Voir par exemple : «ceux que nous avons appelés "poètes" dans notre traduction [de la Poétique] (forts du sens large qui pouvait être appliqué à ce terme [c'est-à-dire qui renvoie à tous ceux qui "façonnent" et pas seulement à ceux qui font de la "poésie"] ), Aristote les appelle littéralement "imitateurs" (mimoumenoi), nous induisant à superposer et à identifier la technique de l'imitation avec celle de la composition ", in Pierre Somville (dir.), Essai sur la Poétique d'Aristote, Vrin, 1975, p. 17.

63. Nous préférons le terme " aspects » plutôt que " données" car les dites données ne sont en réalité jamais « brutes » mais toujours perçues à travers des séries de prismes individuels et collectifs rendus en partie visibles dans la délibération.

64. Anna Jaubert, "Introduction. Cohésion et cohérence : étapes et relais pour l'interprétation ", in Anna Jaubert et Michèle Biraud (dir.), Cohésion et cohérence. Études de linguistique textuelle, ENS Éditions, 2005, p. 8. 
la cohésion habituelle des énoncés, à activer chez le lecteur des cohérences nouvelles, par-delà ses repères courants. Cet exemple montre ainsi qu'en bonne sémiotique de la pertinence, c'est la cohérence qui détermine la cohésion (et non le contraire, comme dans le modèle poétique traditionnel). Il n'y a pas un texte sur lequel projeter plusieurs sens possibles, mais une orientation et une variation de la lecture qui va mettre en forme progressivement un (ou parfois plusieurs) «texte(s) ». Le « texte » produit est le résultat, dans la lecture ordinaire qui combine reconnaissance et délibération, de ces deux dynamiques de la cohérence, celle qui vise à fixer le sens sur des configurations connues (par exemple : je reconnais une actrice connue : c'est une fiction...) et celle qui vise au contraire à « ouvrir » le sens (et si c'était un « documentaire » sur le planning familial, mais en quel sens ?) en explorant plusieurs possibles en fonction des discussions historiquement disponibles et localisées dans le champ social sur les enjeux, les conventions formelles et le statut du simulacre.

Devant la pente glissante de cette notion de texte, nous préférons donc parler de " textualisation », en gardant à l'esprit à travers cette expression que la production d'un " texte ", d'un " tout ", si c'est un enjeu pour le "façonneur » n'en est pas forcément un pour le lecteur/spectateur, comme le montre le plaisir du zapping ou du surf sur le Web. Ainsi, en mettant en rapport deux fragments audiovisuels sans lien a priori pour produire un effet comique, politique ou moral, le Zapping de Canal+ retrouve cette capacité que nous avons à faire $d u$ texte à partir de ce qui nous passe par les yeux et les oreilles, plutôt que un texte. Peu après le 11 septembre 2001, un spectateur peut écrire : " Parler pour ne rien dire, montrer des images en boucle. . . Apparemment, la guerre du Golfe n'a pas servi de leçon ${ }^{65}$, montrant qu'il construit un autre « texte » que celui de la simple vision terrifiante des tours en flammes, alors même qu'elles demeurent sous ses yeux, qu'il relie à un genre, celui de l'actualité en direct. En résumé : il n’y a pas de délibération sans mise en forme(s) de la part du spectateur (par exemple : ces paroles, ce corps sont ceux d'un acteur qui « joue » un personnage, mais je crois percevoir peut-être aussi l'émotion, les sentiments de la personne sous l'acteur) et, dans un mouvement parallèle, le spectateur conduit son enquête sur le sens du film ou du document audiovisuel en donnant de la cohérence aux formes, ce qui produit une mise en cohésion des formes, une " textualisation », plus ou moins aboutie. Du point de vue du jeu des formes, la délibération ne repose donc non pas sur l'identification (reconnaissance du même/du différent) et la totalisation textuelle (retrouver un tout-origine), mais opère par décantation (abductive), jusqu'à produire parfois un questionnement sur la racine du simulacre lui-même, tout en produisant une sédimentation formelle (correspondant à l'historique du parcours, y compris d'un objet à l'autre).

\section{Conclusion}

L'idée d'une délibération des images permet de travailler sur la façon dont les formes audiovisuelles - qu'elles relèvent de la fiction, du documentaire ou d'autres genres et types - sont prises en main par le spectateur au sein de son expérience

65. Courrier des lecteurs de TéléCableSatellite, 6-12 octobre 2001. 
des objets culturels telle qu'on peut la saisir en tant qu'elle est socialisée dans une ou plusieurs discussions publiques. Si elle permet de travailler à davantage de convergence entre les théories de l'espace public, les études de réception et des usages et la sémiotique, elle fait surtout apparaître d'autres configurations formelles que celles que produisent des théories fondées sur le spectateur-modèle ou sur une modélisation a priori, même pragmatique, du spectateur. En partant des réactions attestées de spectateurs, il s'agit de donner sa chance pour l'analyse à cette expérience spectatorielle telle qu'elle est formulée par les spectateurs eux-mêmes. Les grandes organisations du sens (genres, types, formats, conceptions de l'image et de la représentation...) ne disparaissent pas de l'horizon d'analyse, mais elles sont en quelque sorte profilées sous un nouveau jour par la délibération comme décantation, questionnement sur la racine et sédimentation.

On pourrait dire que cette approche " sécularise » d'une certaine manière notre façon de faire de la sémiotique, en particulier, elle se distingue d'une sémiotique de la croyance (du "mythe », de l'" illusion » de la représentation, etc.), issue de Barthes et qui survit dans la mode du "décryptage », où l'analyste vise à dé-mystifier le spectateur. La description la plus forte sans doute de ce régime sémiotique de la croyance a été donnée par Marie-José Mondzain quand elle est remontée aux sources byzantines (le concile de Nicée II) de notre rapport occidental à l'image et des rapports de pouvoirs qui en découlent ${ }^{66}$. La démiurgie auctoriale et ses Weltanschauungen en serait une version post-romantique, mais elle a de bonnes chances de servir de paravent à un phénomène plus prosaïque : la montée du « souci de soi » à travers les catégories esthétiques $^{67}$. Or, cette organisation sociale et religieuse appartient au passé et la sémiotique délibérative montre une tout autre activité du spectateur que celle qui serait régie par cette verticalité et cette clôture d'une communauté de croyants, en tout cas chez les spectateurs occidentaux (ce qui suscite sans doute des tensions accentuées dans d'autres cultures partiellement occidentalisées où les régimes sont confrontés comme à vif). L'usage rhétorique des images et des sons, la disposition critique vis-à-vis des conventions voire des simulacres eux-mêmes témoignent d'une relation bien plus "profane " aux formes audiovisuelles, favorisée par la sécularisation, la pluralisation démocratique des sociétés elles-mêmes, mais aussi, tout simplement, par une pratique généralisée des techniques de l'image et du son. En tout cas, le spectateur contemporain est, sinon incroyant, du moins en tension entre ses habitudes de lecture poétique (qui le conduisent à s'en remettre à un maître du jeu ou du monde, en suivant, sur le mode de la reconnaissance, les grands aiguillages qui organisent les images et les sons) et sa propre capacité délibérative à les interroger et à produire d'autres agencements formels à partir des mêmes images et sons. Peut-être la délibération peut-elle nous aider à passer d'une sémiotique $d u$ profane enfin admise (une sémiotique des objets ordinaires

66. Marie-José Mondzain, Image, icône, économie. Les sources byzantines de l’imaginaire contemporain, Seuil, 1996.

67. C’est l'hypothèse d'Éric Maigret dans «Esthétiques des médiacultures », in Éric Macé et Éric Maigret (dirs), Penser les médiacultures. Nouvelles pratiques et nouvelles représentations du monde, Armand Colin, p. $123-144$. 
de la consommation culturelle) à une sémiotique elle-même profane. Elle permet peut-être aussi de restituer toujours davantage d'agency à ce spectateur ; celui-ci était certes reconnu comme " actif » dans le cadre de la théorie du lecteur-modèle (qui avait besoin de faire une place à cette activité) mais en faisant du lecteur un simple alter ego du démiurge. Le lecteur d'Umberto Eco, capable de puiser dans son « encyclopédie », est un spectateur qui peut mobiliser son "savoir latéral » ${ }^{68}$ sur les images et les sons et coopérer avec le démiurge " tout-sachant », voire entrer dans un rapport de type scientifique et instrumental aux images, dont la version académique est bien représentée par l'ouvrage de Monique Sicard sur La fabrique du regard ${ }^{69}$. Rationnel et non plus religieux, on peut dire que ce spectateur est compétent : spectateur d'après l'empirisme et la Révolution scientifique, il lui manquait peut-être encore d'être reconnu comme capable non seulement de croire et de comprendre, mais aussi de discuter avec les images et les sons. Souvenons-nous qu'il n'y aurait pas eu d'Encyclopédie sans un espace public en gestation. Dans cette perspective, le lecteur n'est plus du tout une figure dédoublée ou comme en miroir de l'auteur-démiurge, car ce dernier n'est pas le seul à avoir un point de vue.

Il est frappant, en tout cas, d'observer comment croyances et compétences sont souvent mises en débat elles-mêmes dans l'élaboration délibérative des spectateurs, précisément en lien avec l'anticipation d'autres positions sociales et d'autres positionnements sémiotiques possibles. Les délibérations spectatorielles nous offrent le portrait d'un spectateur contemporain qui est dans un rapport beaucoup plus matter of fact aux images et aux sons, conscient du rapport de force entre consommateurs et industries du cinéma et de l'audiovisuel ${ }^{70}$, et qui relativise les croyances et les savoirs eux-mêmes en les mettant à l'épreuve de sa propre expérience et des points de vue d'autrui. De même que la sémiotique de la compétence a dynamisé et redimensionné la sémiotique de la croyance, par exemple en suggérant l'existence de spectateurs " avertis » ou " experts », il s'agit avec une sémiotique délibérative de donner une troisième dimension à nos études des formes visuelles et sonores, une dimension qui vienne traverser et interroger sémiotique de la croyance et sémiotique de la compétence pour rapprocher toujours davantage nos modèles et méthodes sémiotiques de la réalité des capacités spectatorielles contemporaines.

\section{Bibliographie}

Vincent Amiel, Esthétique du montage, Nathan, 2001.

Roland Barthes, « Rhétorique de l'image », Communications, 4, 1964, p. 40-51.

Roland Barthes, «La Rhétorique ancienne : aide-mémoire », Communications, 16, 1970, p. 254-340.

David Bordwell et Kristin Thompson, L'art du film : une introduction, De Boeck, 1999.

68. Jean-Marie Schaeffer, op. cit.

69. Monique Sicard, La fabrique du regard. Images de science et appareils de vision $\left(15^{\mathrm{e}}-20^{\mathrm{e}}\right.$ siècle), Odile Jacob, 1998.

70. De nombreux spectateurs sont même conscients des usages commerciaux (marketing) que certaines compagnies de l'Internet peuvent parfois faire de leurs discussions. 
Patrick Charaudeau et Rodolphe Ghiglione (dir.), Paroles en images. Images de paroles. Trois talk-shows européens, Didier Érudition, 1999.

Serge Daney, Devant la recrudescence des vols de sacs à main, cinéma, télévision, information: 1988-1991, Aléas, 1991.

Daniel Dayan, «Le double corps du spectateur », in Jérôme Bourdon et François Jost (dir.), Penser la télévision, Ina-Nathan, 1998, pp. 234-248.

Umberto Eco, Lector in fabula. Ou la coopération interprétative dans les textes narratifs, Grasset, 1985.

Umberto Eco, Les limites de l'interprétation, Le livre de poche, 1994.

Nancy Fraser, «Rethinking the Public Sphere: A Contribution to the Critique of Actually Existing Democracy », Social Text, 25-26, 1990, p. 56-80.

André Gaudreault, Germain Lacasse (dir.), « Le bonimenteur de vues animées/The Moving Picture Lecturer », Iris, 22, Paris/Iowa City, 1996.

Gérard Genette, « La Rhétorique restreinte », Communications, 16, 1970, p. 233-253.

Carlo Ginzburg, Rapports de force. Histoire, Rhétorique, Preuve, Gallimard, 2003.

Robert E. Goodin, «Democratic Deliberation Within », Philosophy \& Public Affairs, 29(1), 2000, pp. 81-109.

Jürgen Habermas, Théorie de l'Agir communicationnel, Fayard, 1987, deux tomes.

Ira Halbertstadt, « An interview with Frederick Wiseman », Filmakers Newsletter, 7(4), 1974.

Stuart Hall, Encoding/Decoding in Television discourse, Archives du Center for Cultural Studies, Stencilled occasional papers, Media series, SP 7, Université Birmingham, 1973.

Stuart Hall, Identités et cultures. Politique des Cultural Studies, Éditions Amsterdam, 2007.

Anna Jaubert, "Introduction. Cohésion et cohérence : étapes et relais pour l'interprétation ", in Anna Jaubert et Michèle Biraud (dir.), Cohésion et cohérence. Études de linguistique textuelle, ENS Éditions, 2005, p. 7-12.

Laurent Jullier et Jean-Marc Leveratto, Cinéphiles et cinéphilie. Une histoire de la qualité cinématographique, Armand Colin, 2010.

Elihu Katz et Tamar Liebes, The Export of Meaning. Cross-Cultural Readings of Dallas, Oxford University Press, 1990.

Jean-Marie Klinkenberg, "Rhétorique de l'argumentation et Rhétorique des figures », in Alain Lempereur et Michel Meyer (dir.), Figures et conflits rhétoriques, Université de Bruxelles, 1990, p. 115-137.

Guy Lochard, «Parcours d'un concept dans les études télévisuelles. Trajectoires et logiques d'emploi », Hermès, 25, "Le dispositif. Entre usage et concept », 1999, p. 143-151.

Guy Lochard et Jean-Claude Soulages, «L'image, faire voir la parole», in Patrick Charaudeau (dir.), La Télévision. Les débats culturels. "Apostrophes », Didier Érudition, 1991.

Éric Maigret, «Esthétiques des médiacultures », in Éric Macé et Eric Maigret (dir.), Penser les médiacultures. Nouvelles pratiques et nouvelles représentations du monde, Armand Colin, p. 123-144.

Marie-José Mondzain, Image, icône, économie. Les sources byzantines de l'imaginaire contemporain, Seuil, 1996.

Chantal Mouffe, The Democratic Paradox, Verso Books, 2000.

Noël Nel, Le débat télévisé, Armand Colin, 1990.

Roger Odin, Cinéma et production de sens, Armand Colin, 1990.

Roger Odin, De la fiction, De Boeck, 2000

Roger Odin, Les Espaces de communication, Presses universitaires de Grenoble, 2011.

Dominique Pasquier, Cultures lycéennes. La tyrannie de la majorité, Autrement, 2005.

Louis Quéré, Les Miroirs équivoques, Aubier, 1982.

François Rastier, "Indices et parcours interprétatifs », in Denis Thouard (dir.), L'interprétation des indices. Enquête sur le paradigme indiciaire avec Carlo Ginzburg, Septentrion, 2007, pp. 123-154. 
Gary Remer, "Two models of deliberation: Oratory and Conversation in Ratifying the Convention ”, Journal of Political Philosophy, 8, 2000, p. 39-64.

Christopher Ricks et Frederick Wiseman, "Language and Documentary Film: A Conversation with Frederick Wiseman », The Threepenny Review, 41, 1990, p. 9-10.

Jean-Marie Schaeffer, L'Image précaire, Seuil, 1987.

Pierre Schaeffer, Machines à communiquer, Seuil, 1970 et 1972, deux tomes.

Geneviève Sellier, La Nouvelle Vague : un cinéma au masculin singulier, CNRS Éditions, 2005.

Monique Sicard, La fabrique du regard. Images de science et appareils de vision $\left(15^{\mathrm{e}}-20^{\mathrm{e}}\right.$ siècle), Odile Jacob, 1998.

Pierre Somville, Essai sur la Poétique d'Aristote, Vrin, 1975.

Guillaume Soulez, "Ethos, énonciation, média. Sémiotique de l'ethos ", Recherches en communication, n 18, Université de Louvain-la-Neuve, 2002, p. 175-198.

Guillaume Soulez, " "Nous sommes le public". Apports de la Rhétorique à l'analyse des publics ", Réseaux, "Figures du public ", vol. XXII, 126, Hermès Publications, 2004, p. 113-141.

Guillaume Soulez, "Rhétorique, public et "manipulation” ", Hermès, 38, CNRS Éditions, 2004, p. 89-95.

Guillaume Soulez, «Parole captée, parole capturée : de Pierre Schaeffer aux années 70 », in François Jost (dir.), Années 70. La télévision en jeu, CNRS Éditions, 2005, p. 107-129.

Guillaume Soulez, "Format et dispositif. Nouvelles règles du jeu esthétique en régime industriel des images et des sons ", in Emmanuelle André, François Jost, Jean-Luc Lioult \& Guillaume Soulez (dir.), Penser la création audiovisuelle. Cinéma, audiovisuel, multimédia, Publications universitaires de Provence, 2009, p. 253-262.

Guillaume Soulez, "L'Algeria tra dialogo e montaggio. Retorica di Muriel (1963) ", in Marina Galetti (dir.), Jean Cayrol, Dalla Notte e Dalla Nebbia, Turin, Kaplan, 2010, p. $258-278$.

Guillaume Soulez, Quand le film nous parle. Rhétorique, cinéma, télévision, PUF, 2011.

Guillaume Soulez, "Les agrégats délibératifs: et s'il n'y avait pas de "communauté" d'interprétation ? La réception délibérative des Bureaux de Dieu par les internautes ", in Marta Boni, Adrienne Boutang, Barbara Laborde et Lucie Merijeau (dir.), Networking images, approches interdisciplinaires des images en réseau, collection Théorème, 17, 2013, p. 119-129.

Janet Staiger, Interpreting Films. Studies in the Historical Receptions of American Cinema, Princeton University Press, 1992.

Eliseo Veron, « De l'image sémiologique aux discursivités. Le temps d'une photo », Hermès, 13-14, 1994, p. 45-64.

\section{GUILLAUME SOULEZ}

